\title{
The near-tip region of a hydraulic fracture with pressure-dependent leak-off and leak-in
}

\author{
Evgenii A. Kanin ${ }^{1}$, Dmitry I. Garagash ${ }^{2,1} \dagger$ and Andrei A. Osiptsov ${ }^{1}$ \\ ${ }^{1}$ Multiphase Systems Lab, Skolkovo Institute of Science and Technology (Skoltech), 3 Nobel \\ Street, Moscow 143026, Russian Federation \\ ${ }^{2}$ Department of Civil and Resource Engineering, Dalhousie University, 1360 Barrington Street, \\ Halifax, Nova Scotia B3H 4R2, Canada
}

(Received $\mathrm{xx}$; revised $\mathrm{xx}$; accepted $\mathrm{xx}$ )

This paper is concerned with an analysis of the near tip region of a propagating fluiddriven fracture in a saturated permeable rock. The study attempts to accurately resolve the coupling between the physical processes - rock breakage, fluid pressure drop in the viscous fluid flow in the fracture, and fluid exchange between fracture and the rock - that exert influence on the hydraulic fracture propagation, yet occur over lengthscales often too small to be efficiently captured in existing coarse grid numerical models. We consider three fluid balance mechanisms: storage in the fracture, pore fluid leak-in from the rock into the fracture as the result of dynamic suction at the dilating crack tip, and fluid leak-off from the fracture into the rock as the fluid pressure in the fracture recovers with distance away from the tip. This process leads to the formation of a pore fluid circulation cell adjacent to the propagating fracture tip. We obtain the general numerical solution for the fracture opening and fluid pressure in the semi-infinite steadily propagating fracture model and fully characterise the solution within the problem parametric space. This allows to identify the parametric regimes of fracture propagation, assess the impact of pore fluid leak-in and the associated near-tip circulation cavity on the solution, and explore limitations of the widely-used, pressure-independent Carter's leak-off model. The obtained solution can be further used as a tip element in a numerical realisation of a solution for a transient growth of a finite fracture (e.g., within the Planar3D approach).

Key words: Boundary layer structure, lubrication theory, porous media, particle/fluid flow

\section{Introduction}

Tensile fracture driven by internal pressurisation of viscous fluid takes place in the transport of magma in the lithosphere (Spence and Turcotte 1985; Lister and Kerr 1991; Rubin 1993), tensile jointing of overpressured saturated rock formation (Secor 1965; Engelder and Lacazette 1990), and in hydraulic fracturing, a widely used method for the development of oil or gas reservoirs. Modelling of these fractures remains a challenge, owing to the strong, non-linear coupling of the governing physical processes, associated with breakage of the rock, viscous fluid flow in the fracture and the fluid exchange between the fracture and permeable formation, often manifested at distances from the moving fracture tip too small to be efficiently resolved in conventional numerical models (e.g.

$\dagger$ Email address for correspondence: garagash@dal.ca 
Detournay (2016) and references therein). To overcome this deficiency, one approach has been to devise an accurate near-fracture-tip model for the small-scale processes (Lister 1990; Desroches et al. 1994; Garagash and Detournay 2000; Garagash et al. 2011), which can then be bridged with the macroscopic process of hydraulic fracture propagation by incorporating it as the near-tip module of an appropriate numerical framework (Siebrits and Peirce 2002; Peirce and Detournay 2008; Dontsov and Peirce 2017; Zia and Lecampion 2018). On both the macro- and micro- scales, the complexity of the fluid flow inside the fracture increases along a number of orthogonal axes (rheology of the carrier fluid, impact of carried proppant particles, bridging of particles, the effect of fracture surface roughness, individual settling or particles and gravitational convection, and interplay between the fluid flow inside the open fracture and outside in the permeable ambient medium) (Osiptsov 2017). In this work we will focus on the classic formulation: Newtonian incompressible fluid, no particles and smooth fracture walls, fracturing and pore fluids have the same properties.

The models of the near-tip region have proliferated since the early contributions focusing on elastohydrodynamic coupling in fully-fluid-filled fracture (Lister 1990; Desroches et al. 1994) to include the effects of the fluid lag and rock fracture toughness (Rubin 1993; Spence and Sharp 1985; Garagash and Detournay 2000), fracturing fluid leak-off into permeable rock (Lenoach 1995; Garagash et al. 2011), pore pressure diffusion and poroelasticity (Detournay and Garagash 2003; Kovalyshen 2010), non-laminar flow in the fracture (Dontsov 2016; Lecampion and Zia 2019), viscous fluid drag onto the fracture walls (Wrobel et al. 2017), and non-Newtonian fracturing fluid rheology (Moukhtari and Lecampion 2018; Dontsov and Kresse 2018), among others.

In this paper we revisit the nature of the fluid exchange between the fracture and the host permeable rock, and its coupling to the fluid flow in the fracture and to the fracture propagation. As the fluid exchange (usually viewed as the leak-off of the pressurized fracturing fluid into the rock) influences the propagating fracture (fluid-filled) volume and the level of fluid pressurisation in the fracture, it exerts the first order influence on the fracture opening and propagation. Fluid exchange between the pressurized fracture and the rock can be complicated by a priori unknown time-and-space varying fluid pressure in the fracture and that of the resulting process of the pore pressure diffusion in the permeable rock, time-dependent poroelastic effects, and the 'cake-building' (deposition of fracturing fluid solids at the fracture walls and in the pore space of the wall-rock). As the result, many modelling attempts resorted to the use of a phenomenological Carter's model (Carter 1957), which suggests that the local rate of fluid exchange (leak-off) at the fracture wall can be approximated by the inverse of the square root of the exposure time (the time since the fracture front has arrived at the considered location along the fracture path). The underpinnings of the Carter's relation is the assumption of the invariant (constant in space and time) fluid pressure differential between the fracture wall and the far field ambient pore pressure in the rock, $p_{f}-p_{o} \approx$ const. The latter assumption often justified on the grounds that the fluid pressure in the fracture scales with the far field confining stress $\sigma_{o}$ (in order for the fracture to stay open), $p_{f} \approx \sigma_{o}$, while the latter assumed to be distinctly larger than the pore fluid pressure, i.e. $\sigma_{o}>p_{o}$, leading to approximately constant pressure differential between the fracture and the rock, $p_{f}-p_{o} \approx \sigma_{o}-p_{o}$. This reasoning, may it be justifiable on average along the fracture length, it does not stand the scrutiny locally when the fluid pressure drop in the flow towards the fracture tip(s) is considered. Indeed, near tip solutions for fully-fracturingfluid-filled hydraulic fracture in impermeable rock (Desroches et al. 1994) and permeable rock with Carter's leak-off (Lenoach 1995; Garagash et al. 2011) lead to the infinite fluid suction at the tip, which not only invalidates the Carter's leak-off assumptions in some 
vicinity of the fracture tip, but actually calls for the separation (lagging) of the fracturing fluid behind the fracture front (Rubin 1993; Garagash and Detournay 2000), and pore fluid leak-in (not fracturing fluid leak-off) into the vacant volume of the (fracturing) fluid lag (Detournay and Garagash 2003). A number of recent numerical studies of the hydraulic fracture propagation in permeable rock which account for the pore pressure diffusion, e.g. (Carrier and Granet 2012; Sarris and Papanastasiou 2011; Golovin and Baykin 2018), do not show pore fluid leak-in, as a possible consequence of the spatially under-resolved fracture tip region in these simulations.

This paper deals with the near-tip region of a fluid-driven fracture propagating in a permeable reservoir rock, while allowing for pressure-dependent fluid leak-off and leakin and associated pore pressure diffusion in the host rock. In formulating the problem, we build on the original model framework of Detournay and Garagash (2003), further generalised by Kovalyshen (2010); Kovalyshen and Detournay (2013). Specifically, we consider the stationary plane-strain problem of a semi-infinite fracture moving at constant speed under the following simplifying assumptions: (i) the fracture propagates under the condition of small scale yielding (or Linear Elastic Fracture Mechanics) (Rice 1968); (ii) the incompressible viscous fracturing fluid is Newtonian and its flow in the fracture is described by Poiseuille lubrication theory (Batchelor 1967); (iii) the fluid exchange between the fracture and the host rock (leak-off and leak-in) is governed by the onedimensional pore pressure diffusion; (iv) possible properties' contrast between the pore and the fracturing fluids is neglected; and (v) the poroelastic 'backstress' effects are considered negligible (Kovalyshen 2010).

This paper is organised as follows. First, the problem formulation, underlining assumptions, and the resulting governing equations are presented. We follow with the discussion of the various asymptotic limits of the solution, including the reduction to the Carter's leak-off case (Garagash et al. 2011), which then allows us to frame the general structure of the sought solution and its parametric dependence. Next, we introduce the characteristic scalings of the solution as they pertain to corresponding limiting regimes of the fracture propagation, and the general non-dimensional problem parametric space defined in terms of two numbers, non-dimensional leak-off $\chi$ and leak-in $\zeta$. The rest of the paper is devoted to the analytical and numerical exploration of the solution to the problem in the parametric space, including an analysis of the relative importance of pressure-dependent effects in the fluid exchange process between the fracture and the reservoir.

\section{Model formulation}

\subsection{Problem definition}

To examine the near tip behaviour of fluid-driven fracture, we consider the problem of a semi-infinite fracture (figure 1) propagating with constant velocity $V$, which is understood as the instantaneous local tip velocity of the parent hydraulic fracture fracture. The host permeable linear-elastic rock is characterised by Young's modulus $E$ and Poisson's ratio $\nu$. Small scale yielding (Rice 1968), i.e. the rock damage/yielding zone at the advancing fracture front small compared to the lengthscales of other physical processes active near the tip (e.g., dissipation in the viscous fluid flow) is assumed. Linear elastic fracture mechanics (LEFM) theory is therefore utilised for the modelling of the quasi-static propagation of the fracture in the rock characterised by the fracture toughness $K_{I c}$.

Figure 1 shows the schematics of the considered problem. The fracture, loaded internally by the fluid pressure $p_{f}(x)$, opens (with aperture distribution $w(x)$ ) against the 


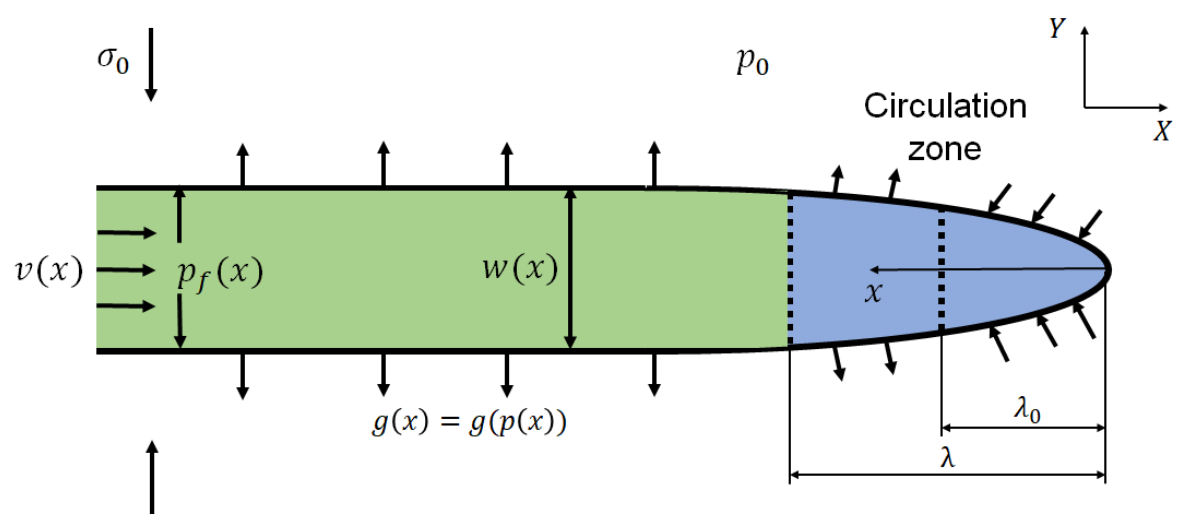

Figure 1: Schematic picture of fracture tip model with pressure-dependent fluid exchange between the fracture and permeable saturated rock.

in-situ confining stress $\sigma_{o}$. We consider fluids presented in the model as Newtonian. Fluid flow in the fracture is described by lubrication theory (Batchelor 1967).

The rock adjacent to the fracture is permeable and saturated by pore fluid at ambient pore pressure $p_{o}$. The fluid exchange between the fracture and the reservoir is driven by the pressure difference between the fracture $\left(p_{f}\right)$ and the reservoir $\left(p_{o}\right)$. The fluid exchange process is modelled by one-dimensional pressure-dependent leak-off/leak-in (PDL) driven by pore pressure diffusion in the rock (Detournay and Garagash 2003; Kovalyshen 2010; Kovalyshen and Detournay 2013). This model is a convenient approximation of a full two-dimensional leak-off and associated diffusion problem (Detournay and Garagash 2003) based on the assumption that the characteristic thickness of the diffusive boundary layer around the crack is small compared to the characteristic lengthscale of the fracture tip problem. The local rate of the fluid exchange is denoted as $g(x)$. We also assume that the pore and hydraulic fracturing fluids have similar (identical in the model) properties.

Fluid pressure $p_{f}(x)$ diminishes in the fluid flow along the fracture towards the tip. If its minimum value at the tip, $p_{f}(0)$, drops below $p_{o}$, there exists a near-tip zone of some length $\lambda_{o}\left(x \in\left[0, \lambda_{o}\right]\right)$ along which the pore fluid flows into the fracture from the surrounding rock. For distance larger than $\lambda_{o}$, the fluid pressure recovers enough to enable the leak-off of the formation fluid from the fracture back into the rock. Due to the steady crack propagation (i.e. problem is stationary in the coordinate system $x$ moving with the crack tip), all of the formation fluid leaked-in along $x \in\left[0, \lambda_{o}\right]$ has to circulate (leak-off) back into the formation, thus defining the pore-fluid circulation zone of some length $\lambda>\lambda_{o}$ near the fracture tip (figure 1). The crack channel withing the interval $[\lambda,+\infty)$ is filled by the hydraulic fracturing fluid which, due to pressure continuity, is also expected to leak-off into the rock.

\subsection{Governing equations}

Let us consider the moving coordinate system $(x, y)$ related to the fixed coordinate system $(X, Y)$ by equations: $x=V t-X, y=Y$. The considered problem is stationary in the moving coordinate system. The governing equations are written for unknown fracture opening $w(x)$ and net pressure distribution $p(x)=p_{f}(x)-\sigma_{o}$ along the fracture $(0<x<+\infty)$, elaborating further on the framework proposed in Garagash et al. (2011). 


\subsubsection{Fracture propagation}

LEFM fracture propagation criteria under quasi-static conditions states that the stress intensity factor at the crack tip matches the rock toughness: $K_{I}=K_{I c}$. This condition prescribes the asymptotic behaviour of the fracture opening near its front (Irvin 1957):

$$
w(x)=\frac{K^{\prime}}{E^{\prime}} \sqrt{x}
$$

where $E^{\prime}=\frac{E}{1-\nu^{2}}$ is the plane strain modulus and $K^{\prime}=4 \sqrt{\frac{2}{\pi}} K_{I c}$ is the toughness parameter.

\subsubsection{Crack elasticity}

The net pressure $p(x)$ in the fracture could be expressed as the crack line integral of the opening $w(x)$ using the elasticity equation (Bilby and Eshelby 1968):

$$
p(x)=\frac{E^{\prime}}{4 \pi} \int_{0}^{\infty} \frac{d w(s)}{d s} \frac{d s}{x-s}
$$

Equation (2.2) can be inverted (Garagash and Detournay 2000) to aid in the numerical implementation of problem solution:

$$
w(x)=\frac{K^{\prime}}{E^{\prime}} \sqrt{x}+\frac{4}{\pi E^{\prime}} \int_{0}^{\infty} K(x, s) p(s) d s
$$

where the integral kernel is: $K(x, s)=\ln \left|\frac{\sqrt{x}+\sqrt{s}}{\sqrt{x}-\sqrt{s}}\right|-2 \sqrt{\frac{x}{s}}$. This form of crack elasticity equation already accounts for the propagation condition (2.1) (i.e. the integral in (2.3) is $o(\sqrt{x}))$.

\subsubsection{Fluid flow}

The flow of viscous incompressible fluid in the crack channel is described by the continuity equation averaged across the fracture aperture, which, upon transforming to the moving coordinate system, is given by:

$$
V \frac{d w}{d x}-\frac{d(w v)}{d x}+g=0
$$

where $g$ is the local rate of fluid exchange between the fracture and the rock $(g>0$ for leak-off and $g<0$ for leak-in) given in the PDL model by the following expression (Appendix A):

$$
g(x)=Q^{\prime} \sqrt{V}\left(\frac{p(0)+\sigma_{o}^{\prime}}{2 \sqrt{x}}+\int_{0}^{x} \frac{d p}{d x^{\prime}} \frac{d x^{\prime}}{2 \sqrt{x-x^{\prime}}}\right)
$$

where $Q^{\prime}=\frac{4 k}{\mu \sqrt{\pi c}}$ is a leak-in coefficient defined in terms of the pore pressure diffusivity coefficient $c$, reservoir permeability $k$ and fluid viscosity $\mu, p(0)=p_{f}(0)-\sigma_{o}$ is the net fluid pressure value at the fracture front, and $\sigma_{o}^{\prime}=\sigma_{o}-p_{o}$ is the ambient value of the effective confining stress.

Integrating Eq. (2.4) from the tip $x=0$ to some distance $x>0$, we obtain:

$$
w v=w V+q_{\perp}, \quad q_{\perp}=\int_{0}^{x} g(s) d s
$$

which signifies that the local fluid volumetric flow rate at distance $x$ from the fracture tip $w(x) v(x)$ is partitioned between the fluid stored in the fracture $w(x) V$ and in the rock via the cumulative rate of fluid exchange $q_{\perp}(x)$, given by 


$$
q_{\perp}(x)=Q^{\prime} \sqrt{V} \int_{0}^{x} \frac{p(s)+\sigma_{o}^{\prime}}{2 \sqrt{x-s}} d s=C^{\prime} \sqrt{V x}+Q^{\prime} \sqrt{V} \int_{0}^{x} \frac{p(s)}{2 \sqrt{x-s}} d s
$$

Here $C^{\prime}=Q^{\prime} \sigma_{o}^{\prime}=\frac{4 k \sigma_{o}^{\prime}}{\mu \sqrt{\pi c}}$ is the Carter's leak-off coefficient. The first term in the right hand side of (2.7) corresponds to the classical Carter's leak-off expression strictly valid only when $p_{f}(x)=\sigma_{o}$ (or $p(x)=0$ ), while the second term is the pressure-dependent correction. Since the net fluid pressure $p(x)<0$ (or $p_{f}(x)<\sigma_{o}$ ) in semi-infinite hydraulic fracture (e.g., Garagash and Detournay (2000)), the corrective pressure-dependent term is always negative, or, in other words, corresponds to a corrective leak-in.

Finally, the Poiseuille's law for the fluid velocity along the crack channel

$$
v=\frac{w^{2}}{\mu^{\prime}} \frac{d p}{d x}
$$

with $\mu^{\prime}=12 \mu$ designating a viscosity parameter, completes the fluid flow description.

\section{Asymptotes and structure of general solution}

\subsection{Vertex solutions}

Two different mechanisms govern the propagation regime of a finite hydraulic fracture, (e.g., Garagash et al. (2011)). The first one is the partitioning of the injected fluid between the fracture and the reservoir as a result of the leak-in and leak-off processes (fracture storage vs fluid exchange with the rock). The second mechanism is partitioning of the total dissipated energy between the creation of the new fracture surfaces and flow of the viscous fluid along the fracture (toughness vs viscosity).

In the process of fracture growth, the partition of the fracturing fluid and the partition of the dissipated energy change over time, which can lead to the realisation of different limiting regimes dominated by one storage and one dissipation mechanisms at different time. In the context of semi-infinite hydraulic fracture, the change in the partitioning of the fluid and of the energy with time can be recast in the change with the distance from the fracture tip.

One can suggest four limiting propagation regimes that are characterised by the dominance of one storage/exchange and one dissipation mechanisms: toughness dominated $\left(\mu^{\prime}=0\right)$, storage-viscosity dominated $\left(C^{\prime}=Q^{\prime}=0, K^{\prime}=0\right)$, leak-off-viscosity dominated $\left(C^{\prime} \rightarrow \infty, K^{\prime}=0\right)$, storage-leak-in-viscosity dominated $\left(K^{\prime}=0, C^{\prime}>0, Q^{\prime}<+\infty\right)$. The corresponding solutions are referred to as "vertex" solutions in a problem parametric space.

While the leak-in $\left(Q^{\prime}\right)$ and the leak-off $\left(C^{\prime}\right)$ coefficients define the partitioning of the fluid, viscosity $\mu^{\prime}$ and toughness $K^{\prime}$ parameters are responsible for the partitioning of dissipated energy.

The first three vertex solutions $(k, m, \widetilde{m})$ are given, e.g., by Garagash et al. (2011), and summarised in table 1 for completeness, in terms of the following three characteristic length scales:

$$
\ell_{k}=\left(\frac{K^{\prime}}{E^{\prime}}\right)^{2}, \ell_{m}=V \frac{\mu^{\prime}}{E^{\prime}}, \ell_{\widetilde{m}}=\left(C^{\prime} \sqrt{V} \frac{\mu^{\prime}}{E^{\prime}}\right)^{2 / 3}
$$

The pressure-dependency of the fluid exchange between the fracture and the rock is coupled with the fluid pressure drop in the flow toward the crack tip (when viscosity is non negligible: $\mu^{\prime}>0$ ). This fact suggests that the leak-in dominates near the fracture front. In other words, we anticipate that near the fracture tip the newly created crack 


\begin{tabular}{lccc}
\hline \multicolumn{1}{c}{ Limiting solutions } & opening $w(x)$ & net pressure $p(x)$ & velocity $v(x)$ \\
\hline $\mathbf{k}$ Toughness $\left(\mu^{\prime}=0\right)$ & $\ell_{k}^{1 / 2} x^{1 / 2}$ & 0 & $V+\frac{E^{\prime} C^{\prime} \sqrt{V}}{K^{\prime}}$ \\
$\mathbf{m}$ Storage-viscosity $\left(K^{\prime}=0, C^{\prime}=Q^{\prime}=0\right)$ & $\beta_{0} \ell_{m}^{1 / 3} x^{2 / 3}$ & $\delta_{0} E^{\prime} \frac{\ell_{m}^{1 / 3}}{x^{1 / 3}}$ & $V$ \\
$\widetilde{\mathbf{m}}$ Leak-viscosity $\left(K^{\prime}=0, C^{\prime} \rightarrow \infty\right)$ & $\widetilde{\beta}_{0} \ell_{\widetilde{m}}^{3 / 8} x^{5 / 8}$ & $\widetilde{\delta}_{0} E^{\prime} \frac{\ell_{m}^{3 / 8}}{x^{3 / 8}}$ & $\frac{V}{\widetilde{\beta}_{0}} \frac{\ell_{m}^{9 / 8}}{\ell_{m} x^{1 / 8}}$ \\
\hline
\end{tabular}

Coefficients: $\beta_{0}=2^{1 / 3} 3^{5 / 6}, \delta_{0}=\beta_{0} f(2 / 3), \widetilde{\beta}_{0}=2.534, \widetilde{\delta}_{0}=\widetilde{\beta}_{0} f(5 / 8)$, with $f(\lambda)=\lambda \cot (\pi \lambda) / 4$

Table 1: Three limiting solutions of a semi-infinite hydraulic fracture for the identified limiting values of problem parameters.

volume (storage) is accommodated entirely by the pore fluid leaking-in from the rock (while the fluid flow towards the fracture tip along the crack channel is negligible there, $v \approx 0$ ). However, this dominance of leak-in has to be limited to a finite near tip region, since crack elasticity requires that $p(x)$ vanishes as $x \rightarrow \infty$, or $p_{f}(x) \rightarrow \sigma_{o}>p_{o}$, thus eventually giving way to the leak-off.

Vertex solutions $k, m, \widetilde{m}$ (table 1 ) provide solution for the entire semi-infinite fracture for the corresponding limiting values of parameters. They can be obtained from the monomial solution to the crack elasticity equation (2.2):

$$
w_{\lambda}(x)=B x^{\lambda} ; p_{\lambda}(x)=E^{\prime} B f(\lambda) x^{\lambda-1}, f(\lambda)=\frac{\lambda \cot (\pi \lambda)}{4}, 0<\lambda<1
$$

where particular values of the prefactor $B$ and the exponent $\lambda$ are constrained by the lubrication equation when setting parameters $\left(C^{\prime}, \mu^{\prime}\right.$ and $\left.K^{\prime}\right)$ to the corresponding limiting values, as detailed by Garagash et al. (2011). In the $k$-vertex, viscosity is negligble $\left(\mu^{\prime}=0\right)$ and solution follows from the propagation condition (2.1). In the $m$-vertex, the fluid exchange $\left(C^{\prime}=Q^{\prime}=0\right)$ and toughness $\left(K^{\prime}=0\right)$ are negligible, and solution is recovered by balancing the fluid flux in the crack $w(x) v(x)$ with the storage term $w(x) V$ in the continuity equation. We anticipate that in the general parametric case (i.e. not limited to the stated values of $K^{\prime}$ and other parameters) the $m$-vertex solution provides the far-field solution asymptote, (e.g., Garagash et al. (2011)). In the $\widetilde{m}$-vertex, the fluid storage $\left(C^{\prime} \rightarrow \infty\right)$, and toughness $\left(K^{\prime}=0\right)$ are negligible. In this case, the fluid flux in the crack is balanced with the Carter's leak-off term. In general case, the $\widetilde{m}$-vertex can be realised as the intermediate field solution (Garagash et al. 2011).

The new storage-leak-in-viscosity vertex $\widetilde{o}$ emerges as a particular case of the viscositydominated $\left(K^{\prime}=0\right)$ behaviour linked to the dominance of the fluid leak-in (rather than the leak-off) in the fracture near field $(x \rightarrow 0)$. It corresponds to the classical zerotoughness behaviour of the crack opening, $w=B_{\widetilde{o}} x^{3 / 2}$, and the non-singular pressure: $p=-\sigma_{0}^{\prime}-\frac{3}{2} B_{\widetilde{o}} \frac{V^{1 / 2}}{Q^{\prime}} x$. The first term in the expression for the net pressure is obtained from balancing the leak-in and leak-off terms in the continuity equation. On the other hand, the second term arises from the matching the leak-in and the fracture storage terms, gains particular importance in/near the zero-leak-off limit $\left(\sigma_{0}^{\prime}=0\right)$. This vertex solution contains prefactor $B_{\widetilde{o}}$ (with units $1 / \sqrt{m}$ ) that is unknown and a part of the overall solution. This betrays the fact that the $\widetilde{o}$-asymptote can only be realised as the near or intermediate field of the fracture, as it can not satisfy the elasticity equation over 
opening $w(x)$ net pressure $p(x)$ velocity $v(x)$

\begin{tabular}{|c|c|c|c|}
\hline $\mathbf{k}$ Toughness $\left(K^{\prime}>0\right)$ & $\ell_{k}^{1 / 2} x^{1 / 2}$ & $-\sigma_{0}^{\prime}-\frac{K^{\prime} V^{1 / 2}}{E^{\prime} Q^{\prime}}$ & 0 \\
\hline$\widetilde{\mathbf{o}}$ Storage-leak-in-viscosity $\left(K^{\prime}=0\right)$ & $B_{\widetilde{o}} x^{3 / 2}$ & $-\sigma_{0}^{\prime}-\frac{3}{2} B_{\widetilde{o}} \frac{V^{1 / 2}}{Q^{\prime}} x$ & $-\frac{3}{2} B_{\widetilde{o}}^{3} \frac{V^{1 / 2}}{Q^{\prime} \mu^{\prime}} x^{3}$ \\
\hline
\end{tabular}

Coefficients: $B_{\widetilde{o}}$ is a part of the solution.

Table 2: Near-field $(x \rightarrow 0)$ of semi-infinite hydraulic fracture

the full semi-infinite crack extent. The second term in the net pressure is found with the assumption that the $\widetilde{o}$-vertex solution is realised in the near-field, and in this case, the left-hand side of the continuity equation $\left(\sim w^{3}(x) p^{\prime}(x)\right)$ for this vertex solution is negligible as compared to terms in the right-hand side (storage, leak-off and leak-in).

For non-zero fracture toughness $K^{\prime}>0$, the near-field $(x \rightarrow 0)$ behaviour of the fracture opening is given by the $k$-vertex solution (Table 1 ), as stems from the propagation condition (2.1). Corresponding asymptotic expression for the net pressure $p(x \rightarrow$ $0)=-\sigma_{0}^{\prime}-\frac{K^{\prime} V^{1 / 2}}{E^{\prime} Q^{\prime}}$ follows from the fluid continuity equation (2.6) by balancing the fluid exchange (the leak-in pore fluid volume) with the fracture storage. (We note that the fluid flux along the crack $w v$ is negligibly small in the near field fluid balance). The obtained finite net pressure value at the fracture tip is drastically different from the one in the Carter's, pressure-independent leak-off model (Garagash et al. 2011), where the pressure sustains a negative singularity as the fracturing fluid is assumed to reach the tip of the fracture. When reformulated in terms of the fluid pressure, $p_{f}(x \rightarrow 0)=p_{0}-K^{\prime} \sqrt{V} /\left(E^{\prime} Q^{\prime}\right)$, this asymptote suggests that the fluid pressure at the crack tip is reduced from its drained value given by the ambient pore pressure $p_{0}$ by the amount $K^{\prime} \sqrt{V} /\left(E^{\prime} Q^{\prime}\right)$. The latter, undrained pressure change vanishes for slowly propagating cracks $(V \rightarrow 0)$ or/and zero rock toughness $\left(K^{\prime} \rightarrow 0\right)$.

The obtained near-field $k\left(K^{\prime}>0\right)$ and $\widetilde{o}\left(K^{\prime}=0\right)$ asymptotes are summarised in table 2 .

\subsection{Structure of solution and scaling}

The general solution of the considered problem can be tracked within the parametric triangular pyramid $m \tilde{m} \widetilde{o} k$ formed by four aforesaid vertices. The schematic picture of this pyramid is represented in figure 2 .

Four triangular faces of pyramid $m \widetilde{m} \widetilde{o} k$ correspond to either the dominance of one of the three fluid storage/exchange mechanisms or one of the dissipation mechanisms:

- storage-leak-off face $m \tilde{m} k$ : leak-in process is negligible, $Q^{\prime}=0$

- storage-leak-in face $m \widetilde{o} k$ : leak-off process is negligible, $C^{\prime} \propto \sigma_{o}^{\prime}=0$

- leak-face $\widetilde{m} \widetilde{o}$ : fluid storage in the fracture is negligible, $C^{\prime} \rightarrow \infty$

- viscosity-face $m \widetilde{m} \widetilde{o}$ : toughness is negligible, $K^{\prime}=0$

Six edges of the pyramid $m \widetilde{m} \widetilde{o} k$ correspond to intersection of the corresponding two faces, and thus, reflect the dominance of one of the three fluid storage/exchange mechanisms and one of the dissipation mechanisms. E.g., $\widetilde{m} \widetilde{o}$ is the leak-viscosity edge $\left(C^{\prime} \rightarrow \infty\right.$ and $\left.K^{\prime}=0\right)$, bounding the leak $\widetilde{m} \widetilde{o} k\left(C^{\prime} \rightarrow \infty\right)$ and the viscosity $m \widetilde{m} \widetilde{o}\left(K^{\prime}=0\right)$ faces, and, thus, corresponds to the negligible storage and toughness.

Proposed pyramidal parametric space $m \widetilde{m} \widetilde{o} k$ for the HF with pressure-dependent leak- 


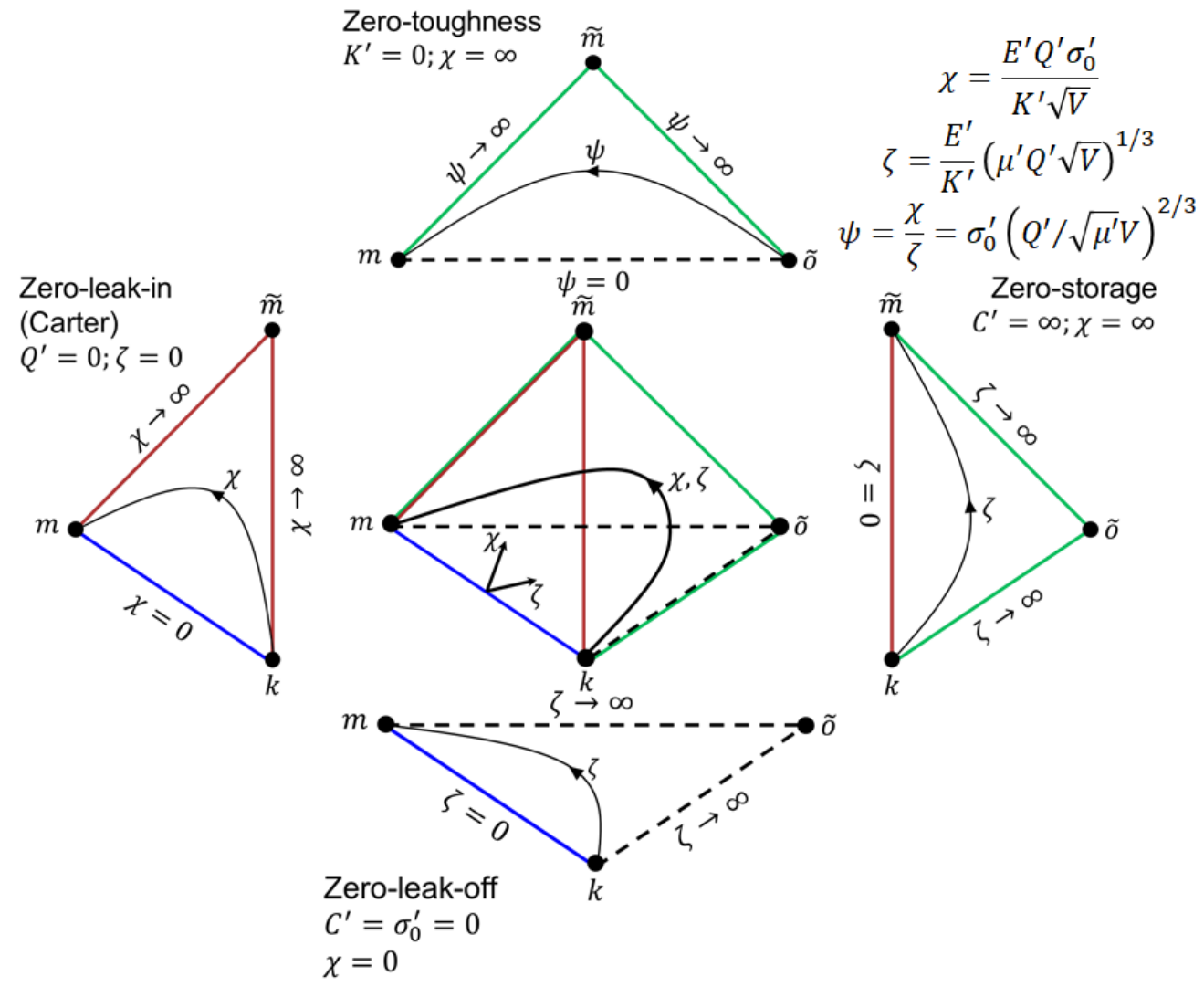

Figure 2: Parametric diagram (pyramid $m \tilde{m} \widetilde{o} k$ ) and corresponding four limiting faces corresponding to the dominance of one energy dissipation or one fluid storage mechanism. Few solution trajectories parameterized by the leak-off $\chi$ and leak-in $\zeta$ numbers (or their ratio $\psi=\chi / \zeta)$ are also shown.

off is a direct generalization of the triangular parametric space, face $m \widetilde{m} k$, for the Carter's (pressure-indepdent) leak-off (Garagash et al. 2011), by the addition of the new vertex $\widetilde{o}$. The emergent edges $\widetilde{o} k, \widetilde{m} \widetilde{o}$, and $m \widetilde{o}$ are expected to describe the transitions of the corresponding limiting solutions with distance from the crack tip between corresponding vertices (from the $2^{\text {nd }}$ to the $1^{\text {st }}$, i.e. $\widetilde{m} \widetilde{o}$-edge corresponds to the transition from the near-field $\widetilde{o}$ to the far-field $\widetilde{m}$, etc). As discussed in the above, the $\widetilde{o}$-vertex solution can only be realised in the near-field of a semi-infinite fracture, thus, suggesting that the $\widetilde{o} k$-edge may in fact corresponds to the near-field expansion of the $k$-vertex $\left(w \propto x^{1 / 2}\right)$ which includes the next order correction given by $\widetilde{o}$-vertex solution $\left(w \propto x^{3 / 2}\right)$, which may come to eventually dominate (over the $k$-term) with increasing distance from the tip.

In the case of the other two edges involving the $\widetilde{o}$-vertex as the fracture near-field, i.e. $\widetilde{m} \widetilde{o}\left(K^{\prime}=0, C^{\prime} \rightarrow \infty\right)$ and $m \widetilde{o}\left(K^{\prime}=0, C^{\prime} \propto \sigma_{o}^{\prime}=0\right)$, they should in principle provide solutions for the entire semi-infinite HF under the corresponding limiting values of the parameters. Before attempting these (and other edge) solutions, let us attempt to constrain the apriori unknown near-field coefficient $B_{\widetilde{o}}$ in the $\widetilde{o}$ expression for the opening $w=B_{\widetilde{o}} x^{3 / 2}(x \rightarrow 0)$. Using the inverted form of the elasticity equation (2.3) 
with $K^{\prime}=0$, and formally passing to the asymptotic limit $x \rightarrow 0$ under the integral, we get

$$
B_{\widetilde{o}}=\frac{8}{3 \pi E^{\prime}} \int_{0}^{\infty} \frac{p(s)}{s^{3 / 2}} d s
$$

Since $p(s \rightarrow 0)=-\sigma_{0}^{\prime}-\frac{3}{2} B_{\widetilde{o}} \frac{V^{1 / 2}}{Q^{\prime}} s$ (Table 3 ), the above integral expression for $B_{\widetilde{o}}$ converges (finite) for the $m \widetilde{o}$-edge (when $\sigma_{o}^{\prime}=0$ ) and diverges for the $\widetilde{m} \widetilde{o}$-edge. This suggests that the underlining formal limit-taking procedure to arrive to (3.2) is not applicable to the latter ( $\widetilde{m} \widetilde{o}$-edge), while conversely $(3.2)$ can be used to constrain coefficient $B_{\widetilde{o}}$ in the former case $(m \widetilde{o}$-edge). Specifically, we observe for the $m \widetilde{o}$-edge that if the net pressure is negative in the entire crack coordinate domain $(p(s)<0$ for all $s>0$ ), which is suggested by the negative net pressure values in the both near and far fields, then (3.2) results in $B_{\widetilde{o}}<0$ or, in other words, negative crack opening near the tip. This contradiction rules out the existence of the $m \widetilde{o}$-edge solution (under plausible assumption of the negative net pressure in the crack), which implies that the general solution to the problem does not have well-defined limiting solution when both toughness and leak-off (or, conversely, ambient effective stress) are null.

General solution of the fracture tip problem within the parametric pyramid transitions with increasing distance from the tip from the near field $k$ to the far field $m$ vertex, and in different limiting cases can collapse onto or be attracted to a series of faces and/or edges, as apparent from their parametric definitions. To identify non-dimensional parameters which fix a given solution trajectory in the parametric space, we follow the methodology of Garagash et al. (2011), and introduce characteristic scales for the transition distance $\ell_{*}$, opening $w_{*}$, and pressure $p_{*}$ closely related to the evolution of the solution along a given edge in the parametric space between the two corresponding vertices, or the 'edge-scalings'.

Edge-scaling $m k, \tilde{m} k$, and $m \tilde{m}$ are defined after Garagash et al. (2011) such that the solutions for either $p(x)$ or $w(x)$ for the corresponding two vertices forming the edge in question "intersect" at $x \sim \ell_{*}$. For example, in the case of the storage $m k$ edge, we find the characteristic length by contrasting the $k$ and $m$ asymptotes for the opening, $w_{*}=\ell_{k}^{1 / 2} \ell_{*}^{1 / 2}=\ell_{m}^{1 / 3} \ell_{*}^{2 / 3}$, while $p_{*}$ follows from the elastic scaling constraint $w_{*} / \ell_{*}=p_{*} / E^{\prime}$. Edge-scalings which involve vertex $\widetilde{o}$ (i.e. $\widetilde{m} \widetilde{o}$ and $\widetilde{o} k$ ) are obtained similarly but also recognising that the $\widetilde{o}$-asymptote depends on the solution trajectory (via a-priori unknown prefactor). In the $\widetilde{m} \widetilde{o}$-case, the transition lengthscale $\ell_{*}$ is found by contrasting the leading order $\widetilde{o}$-asymptote for the net pressure (i.e. $p \approx-\sigma_{o}^{\prime}$ ) with that of the $\widetilde{m}$-vertex, i.e. $p_{*}=\sigma_{o}^{\prime}=E^{\prime}\left(\ell_{\widetilde{m}} / \ell_{*}\right)^{3 / 8}$, while $w_{*}$ follows from the elastic constraint. In the $\widetilde{o} k$-scaling the characteristic pressure is taken equal to $p_{*}=\sigma_{0}^{\prime}$. Using $p_{*}$, elastic scaling constraint and balancing $\widetilde{o}$ and $k$ vertex solutions, we find $\ell_{*}=K^{\prime 2} / \sigma_{o}^{\prime 2}$ and $w_{*}=K^{\prime 2} / E^{\prime} \sigma_{o}^{\prime}$. All of the above edge-scalings are recorded in Table 3 .

Comparing three transition (edge) lengthscales within a given parametric face of the pyramid $m \widetilde{m} \widetilde{o} k$ allows to identify a 'trajectory number' parameterising that face solution. Considering, for example, the zero-leak-in face $m \tilde{m} k, \zeta=0$, one can introduce a single number expressible as a ratio of any two of the this face's three transition lengthscales $\left(\ell_{m k}, \ell_{m \widetilde{m}}\right.$, and $\left.\ell_{\widetilde{m} k}\right)$ (Garagash et al. 2011)

$$
\chi=\left(\frac{\ell_{m \widetilde{m}}}{\ell_{m k}}\right)^{1 / 6}=\left(\frac{\ell_{m k}}{\ell_{\widetilde{m} k}}\right)^{1 / 2}=\left(\frac{\ell_{m \widetilde{m}}}{\ell_{\widetilde{m} k}}\right)^{1 / 8}=\frac{C^{\prime} E^{\prime}}{K^{\prime} V^{1 / 2}}
$$

This number, which can be interpreted as a dimensionless leak-off or ambient effective stress (since $C^{\prime}=Q^{\prime} \sigma_{o}^{\prime}$ ), or non-dimensional reciprocal of toughness, parametrises 


\begin{tabular}{llll} 
Scaling & \multicolumn{1}{c}{$\ell_{*}$} & \multicolumn{1}{c}{$w_{*}$} & \multicolumn{1}{c}{$p_{*}$} \\
\hline$m k$ & $\ell_{m k}=\ell_{k}^{3} / \ell_{m}^{2}$ & $\ell_{k}^{2} / \ell_{m}$ & $E^{\prime} \ell_{m} / \ell_{k}$ \\
$m \widetilde{m}$ & $\ell_{m \widetilde{m}}=\ell_{\widetilde{m}}^{9} / \ell_{m}^{8}$ & $\ell_{\tilde{m}}^{6} / \ell_{m}^{5}$ & $E^{\prime}\left(\ell_{m} / \ell_{\tilde{m}}\right)^{3}$ \\
$\widetilde{m} k$ & $\ell_{\widetilde{m} k}=\ell_{k}^{4} / \ell_{\widetilde{m}}^{3}$ & $\ell_{k}^{5 / 2} / \ell_{\widetilde{m}}^{3 / 2}$ & $E^{\prime}\left(\ell_{\widetilde{m}} / \ell_{k}\right)^{3 / 2}$ \\
$\widetilde{m} \widetilde{o}$ & $\ell_{\tilde{m} \widetilde{o}}=\left(E^{\prime} / \sigma_{o}^{\prime}\right)^{8 / 3} \ell_{\widetilde{m}}$ & $\left(E^{\prime} / \sigma_{0}^{\prime}\right)^{5 / 3} \ell_{\widetilde{m}}$ & $\sigma_{0}^{\prime}$ \\
$\widetilde{o} k$ & $\ell_{\tilde{o} k}=\left(K^{\prime} / \sigma_{0}^{\prime}\right)^{2}$ & $K^{\prime 2} /\left(E^{\prime} \sigma_{0}^{\prime}\right)$ & $\sigma_{0}^{\prime}$ \\
\hline
\end{tabular}

Table 3: Characteristic distance from the tip $\ell_{*}$, pressure $p_{*}$, and opening $w_{*}=\left(p_{*} / E^{\prime}\right) \ell_{*}$, corresponding to the five scalings of the problem.

solution trajectory within the $m \tilde{m} k$-face. The limiting case $\chi \rightarrow 0$ corresponds to the storage-dominated $m k$-edge solution

$$
\zeta=0, \chi=0: \quad k \underset{\ell_{m k}}{\rightarrow} m
$$

which transitions from the $k$ to the $m$ vertex with distance from the tip over lengthscale $\ell_{m k}$ (shown by blue-colour trajectory in figure 2). While the other limiting case $\chi \rightarrow \infty$ corresponds to the separation of the corresponding transitional scales, $\ell_{\widetilde{m} k} \ll \ell_{m \widetilde{m}}$, (3.3), leading to the nested solution structure corresponding to the succession of the two edge solutions $(\widetilde{m} k$ and $m \tilde{m})$

$$
\zeta=0, \chi \rightarrow \infty: \quad k \underset{\ell_{\widetilde{m} k}}{\rightarrow} \widetilde{m} \underset{\ell_{m \widetilde{m}}}{\rightarrow} m
$$

signifying transition with distance from the tip first from the $k$ to $\widetilde{m}$ vertex over lengthscale $\ell_{\widetilde{m} k}$ and then from the $\widetilde{m}$ to $m$ vertex over lengthscale $\ell_{m \widetilde{m}}$ (shown by browncolour trajectory in figure 2).

Similarly, for the zero-storage face $\widetilde{m} \widetilde{o} k, \chi \rightarrow \infty$, we define another number in terms of ratios of any two of the corresponding three edge lengthscales $\left(\ell_{\widetilde{o} k}, \ell_{\widetilde{m} \widetilde{o}}\right.$, and $\left.\ell_{\widetilde{m} k}\right)$

$$
\zeta=\left(\frac{\ell_{\widetilde{o} k}}{\ell_{\widetilde{m} k}}\right)^{1 / 6}=\left(\frac{\ell_{\widetilde{m} \widetilde{o}}}{\ell_{\widetilde{o} k}}\right)^{1 / 2}=\left(\frac{\ell_{\widetilde{m} \widetilde{o}}}{\ell_{\widetilde{m} k}}\right)^{1 / 8}=\frac{E^{\prime}}{K^{\prime}}\left(\mu^{\prime} Q^{\prime} V^{1 / 2}\right)^{1 / 3}
$$

which can be interpreted as dimensionless leak-in or a reciprocal of toughness. This number parametrises solution trajectory within the $\widetilde{m} \widetilde{o} k$-face. The limiting case $\zeta \rightarrow$ 0 corresponds to the leak-off-dominated $\widetilde{m} k$-edge solution, also a part of the limiting trajectory (3.5) in the $m \tilde{m} k$ face. While $\zeta \rightarrow \infty$ corresponds to the separation of the relevant transitional scales, $\ell_{\widetilde{o} k} \ll \ell_{\widetilde{m} \widetilde{o}}$, (3.6), leading to the nested solution structure corresponding to the succession of the two edge solutions ( $\widetilde{o} k$ and $\widetilde{m} \widetilde{o})$ with distance from the tip

$$
\chi \rightarrow \infty, \zeta \rightarrow \infty: \quad k \underset{\ell_{\tilde{o} k}}{\rightarrow} \widetilde{o} \underset{\ell_{\tilde{m} \tilde{o}}}{\rightarrow} \widetilde{m}
$$

For the the zero-toughness face $m \widetilde{m} \widetilde{o}, \chi \rightarrow \infty$, we can define another number in terms of ratio of the $\widetilde{m} \widetilde{o}$ and $m \widetilde{m}$ edge lengthscales

$$
\psi=\left(\frac{\ell_{m \widetilde{m}}}{\ell_{\widetilde{m} \widetilde{o}}}\right)^{1 / 8}=\sigma_{o}^{\prime}\left(\frac{Q^{\prime}}{\mu^{\prime 1 / 2} V}\right)^{2 / 3}
$$




\begin{tabular}{|c|c|c|c|}
\hline Scaling & Lubrication (2.6)-(2.8) & Elasticity (2.2) & $\underset{\xi \rightarrow 0}{\operatorname{Propagation}}(2.1)$ \\
\hline$m k$ & $\Omega^{3} \frac{\mathrm{d} \Pi}{\mathrm{d} \xi}=\Omega+\chi \xi^{1 / 2}+\zeta^{3} \int_{0}^{\xi} \frac{\Pi(s) d s}{2 \sqrt{\xi-s}}$ & & $\Omega=\xi^{1 / 2}$ \\
\hline$m \tilde{m}$ & $\Omega^{3} \frac{\mathrm{d} \Pi}{\mathrm{d} \xi}=\Omega+\xi^{1 / 2}+\psi^{-3} \int_{0}^{\xi} \frac{\Pi(s) d s}{2 \sqrt{\xi-s}}$ & & $\Omega=\chi^{-1} \xi^{1 / 2}$ \\
\hline$\widetilde{m} k$ & $\Omega^{3} \frac{\mathrm{d} \Pi}{\mathrm{d} \xi}=\chi^{-1} \Omega+\xi^{1 / 2}+\zeta^{3} \int_{0}^{\xi} \frac{\Pi(s) d s}{2 \sqrt{\xi-s}}$ & $\Pi(\xi)=\frac{1}{4 \pi} \int_{0}^{\infty} \frac{d \Omega(s)}{\xi-s}$ & $\Omega=\xi^{1 / 2}$ \\
\hline$\tilde{m} \widetilde{o}$ & $\Omega^{3} \frac{\mathrm{d} \Pi}{\mathrm{d} \xi}=\psi^{-1} \Omega+\xi^{1 / 2}+\int_{0}^{\xi} \frac{\Pi(s) d s}{2 \sqrt{\xi-s}}$ & & $\Omega=\zeta^{-1} \xi^{1 / 2}$ \\
\hline$\widetilde{o} k$ & $\zeta^{-3} \Omega^{3} \frac{\mathrm{d} \Pi}{\mathrm{d} \xi}=\chi^{-1} \Omega+\xi^{1 / 2}+\int_{0}^{\xi} \frac{\Pi(s) d s}{2 \sqrt{\xi-s}}$ & & $\Omega=\xi^{1 / 2}$ \\
\hline
\end{tabular}

Table 4: Normalised governing equations for the scaled opening $\Omega=w / w_{*}$ and net pressure $\Pi=p / p_{*}$ as a function of the scaled position $\xi=x / \ell_{*}$ in different scalings $\left(\ell_{*}, w_{*}, p_{*}\right)$ from table 3 .

which can be interpreted as, e.g., dimensionless effective confining stress. Note that $\psi$ is not an independent parameter, but expressible in terms of the previously introduced leak-off $\chi$ and leak-in $\zeta$ numbers, $\psi=\chi / \zeta$. This number parametrises solution trajectory within the $m \tilde{m} \widetilde{o}$ face, such that $\psi \rightarrow \infty$ corresponds to the separation of the two lengthscales, $\ell_{\tilde{m} \widetilde{o}} \ll \ell_{m \widetilde{m}}$, (3.8), resulting in the solution comprised of the two edge solutions ( $m \widetilde{o}$ and $m \widetilde{m})$

$$
\chi \rightarrow \infty, \psi=\chi / \zeta \rightarrow \infty: \quad \widetilde{o} \underset{\ell_{\widetilde{m} \widetilde{o}}^{\rightarrow}}{\rightarrow} \underset{\ell_{m \widetilde{m}}}{\rightarrow} m
$$

The other limit, $\psi=0$, corresponding to the viscosity-leak-in $m \widetilde{o}$ edge, is not expected to exist per discussion in the above. The behaviour of the solution within the $m \widetilde{m} \widetilde{o}$ and particularly how it approaches the non-existing $m \widetilde{o}$ edge with diminishing value of $\psi$ is to be explored numerically.

We note that in the case when the parametric conditions in (3.7) and (3.9) are combined, i.e. when $\chi \rightarrow \infty, \zeta \rightarrow \infty$, and $\psi=\chi / \zeta \rightarrow \infty$, the three scales separate, $\ell_{\widetilde{o} k} \ll \ell_{\widetilde{m} \widetilde{o}} \ll \ell_{m \widetilde{m}}$, and the 'triple-nested' solution structure is realised

$$
\chi \rightarrow \infty, \zeta \rightarrow \infty, \psi=\chi / \zeta \rightarrow \infty: \quad k \underset{\ell_{\tilde{o} k}}{\rightarrow} \widetilde{o} \underset{\ell_{\tilde{m} \tilde{o}}^{\rightarrow}}{\rightarrow} \widetilde{\ell_{m \widetilde{m}}} \rightarrow m
$$

as shown by the green-colour trajectory in figure 2 .

For the fourth and final face of the pyramid, the zero-leak-off face $m \widetilde{o} k, \chi=0$, we can use the previously defined non-dimensional leak-in number $\zeta$ to track solution trajectories, such that $\zeta=0$ corresponds to the storage $m k$-edge, and $\zeta \rightarrow \infty$ corresponds to the non-existing limit of either $m \widetilde{o}$ edge or the $\widetilde{o} k$ edge. (Note that the $\widetilde{o} k$ edge can only be realised as the near or near-to-intermediate field of a given solution, thus, nonexistence of the $\widetilde{o} m$ solution, which would form the intermediate-to-far-field solution in the limit $\zeta \rightarrow \infty$, implies the non-existence of the near-to-intermediate-field, $\widetilde{o} k$ edge, solution within the zero-leak-off face $m \widetilde{o} k$ ). 


\subsection{Asymptotic expansions of the vertices}

Some insight into how the solution departs from the vertices in the parametric space in response to small perturbation of problem parameters and distance from the fracture tip can be afforded by constructing corresponding asymptotic expansions.

\subsubsection{Expansion near $k$-vertex}

The near-field $k$-vertex expression (table 2) for the net pressure is simply given by the value at tip set by the balance between incipient fluid exchange and crack opening, respectively, and thus independent of the fluid flow along the crack channel. The latter becomes more important when moving away from the tip, and can be accounted for by incorporating next order terms in the $k$-vertex asymptotic expansion (Appendix B). The $k$-expansion for net fluid pressure is given by

$$
\begin{array}{rlrl}
\zeta>0: & \frac{p}{E^{\prime}} & =\frac{\ell_{k}^{1 / 2}}{\ell_{1}^{1 / 2}}\left[-\frac{1}{\zeta^{3}}+\frac{1}{\gamma(\zeta)}\left(\frac{x}{x_{o}}\right)^{\gamma(\zeta)}\right] \\
\zeta=0: & \frac{p}{E^{\prime}}=\frac{\ell_{k}^{1 / 2}}{\ell_{1}^{1 / 2}} \ln \left(\frac{x}{x_{o}}\right)
\end{array}
$$

in the general $\zeta>0$ case and in the Carter's $\zeta \rightarrow 0$ limit (Garagash et al. 2011), respectively. Lengthscale $\ell_{1}$ is defined in terms of a pair of transitional lengthscales

$$
\ell_{1}=\left(\ell_{m k}^{-1 / 2}+\ell_{\widetilde{m} k}^{-1 / 2}\right)^{-2}
$$

Exponent $\gamma=\gamma(\zeta)$ in (3.11) is given implicitly by (B 3), and $x_{o}$ is a priori unknown part of the solution. One can directly confirm that (3.11) reduces to (3.12) in the Carter's limit $\zeta \rightarrow 0$ in view of the vanishing power-law exponent $\gamma(\zeta \rightarrow 0) \sim \zeta^{3}$.

We again point out the marked difference in the net pressure behaviour near the fracture tip between the general (pressure dependent leak-off case $\zeta>0$ ) and the Carter's limit. In the former, the net pressure at the tip is bounded $p(0)=-E^{\prime}\left(\ell_{k} / \ell_{0}\right)^{1 / 2}=$ $-\left(\sigma_{o}^{\prime}+K^{\prime} V^{1 / 2} / E^{\prime} Q\right)$, while in the latter - logarithmically singular.

Corresponding $k$-vertex expansion for the crack opening is

$$
\begin{array}{ll}
\zeta>0: & w=\ell_{k}^{1 / 2} x^{1 / 2}+\frac{\ell_{k}^{1 / 2}}{\ell_{1}^{1 / 2}}\left[\frac{4 \tan \pi \gamma}{\gamma(1+\gamma)} \frac{x^{\gamma+1}}{x_{o}^{\gamma}}+\frac{x^{3 / 2}}{x_{1}^{1 / 2}}\right] \\
\zeta=0: & w=\ell_{k}^{1 / 2} x^{1 / 2}+\frac{\ell_{k}^{1 / 2}}{\ell_{1}^{1 / 2}} 4 \pi x
\end{array}
$$

where $x_{o}$ and $x_{1}$ are a priori unknown parts of the solution. Once again, the Carter's expression (3.14), identical to that of (Garagash et al. 2011) follows from the general expression (3.13) when taking the limit $\zeta \rightarrow 0$ in the latter. We observe that the choice of the next order term (after the leading term $\sim x^{1 / 2}$ ) in the opening expansion depends on the value of $\gamma(\zeta)$. Specifically, it is given by $x^{\gamma+1}$ term when $\gamma(\zeta)+1<3 / 2$, corresponding to $\zeta<0.862$, and by the $x^{3 / 2}$ term otherwise (when $\zeta>0.862$ ). In relation to the problem parametric diagram, the $\zeta$-dependent form of the next order term in (3.14) determines how the solution trajectory emanates from the $k$-vertex along a given $\zeta$ trajectory. For example, considering the zero-storage $\widetilde{m} \widetilde{o} k$ face, the zero-leak-in $(\zeta=0)$ trajectory exits the $k$-vertex along the $\widetilde{m} k$-edge described by the linear correction $x^{\gamma(0)+1}(\gamma(0)=0)$ to the opening, and the zero-leak-off $(\zeta=\infty)$ trajectory exits the $k$-vertex along the $\widetilde{o} k$-edge described by the $x^{3 / 2}$ correction to the opening. 


\subsubsection{Expansion near $m$-vertex}

The $m$-vertex solution does not depend on the rock toughness and the parameters defining fluid-exchange processes. Moving away from the far-field region, where the $m$ vertex dominates, towards the crack tip, the latter effects start to influence the solution. These higher-order effects can be captured in the $m$-vertex expansion which can be obtained using the procedure of Garagash et al. (2011) (thier Appendix C) in the following form:

$$
w=\ell_{m}^{1 / 3} x^{2 / 3}\left[\beta_{0}+\sum_{j=1}^{3} \beta_{-j}\left(\frac{\ell_{m \widetilde{m}}}{x}\right)^{j / 6}+\stackrel{\circ}{\beta}-3^{\left(\frac{\ell_{m \widetilde{o}}}{x}\right)^{1 / 2}}+\underline{\left.\underline{\left(\beta_{-4}\right)_{*}(\chi, \zeta)\left(\frac{\ell_{m *}}{x}\right)^{2 / 3-h}}\right]}\right]
$$

$\frac{p}{E^{\prime}}=\ell_{m}^{1 / 3} x^{-1 / 3}\left[\delta_{0}+\sum_{j=1}^{3} \delta_{-j}\left(\frac{\ell_{m \widetilde{m}}}{x}\right)^{j / 6}+\stackrel{\circ}{\delta}-3^{\left(\frac{\ell_{m \widetilde{o}}}{x}\right)^{1 / 2}}+\underline{\left.\underline{\left(\delta_{-4}\right)_{*}(\chi, \zeta)\left(\frac{\ell_{m *}}{x}\right)^{2 / 3-h}}\right]}\right.$

where coefficients $\beta_{-j}$ are

$$
\beta_{0}=2^{1 / 3} 3^{5 / 6}, \beta_{-1}=\frac{1}{2}, \beta_{-2}=-\frac{3^{1 / 6}}{2^{7 / 3}}, \beta_{-3}=\frac{2^{7 / 3}}{3^{5 / 3}}, \stackrel{\circ}{\beta}_{-3}=-\frac{2^{7 / 3}}{3^{5 / 3}} \frac{9 \Gamma\left(\frac{2}{3}\right) \Gamma\left(\frac{5}{6}\right)}{2 \sqrt{\pi}}
$$

and $\delta_{-j}=\beta_{-j} f\left(\frac{2}{3}-\frac{j}{6}\right)$ for $j=0,1,2,3, \stackrel{\circ}{\delta}_{-3}=\stackrel{\circ}{\beta}_{-3} f(7 / 6),\left(\delta_{-4}\right)_{*}=\left(\beta_{-4}\right)_{*} f(h)$ with $h=0.138673$.

The terms in Eq. (3.15) and (3.16) underlined by a single line are scaled by characteristic length $\ell_{m \widetilde{m}}$ and represent leak-off corrections to the $m$-vertex solution. The term underlined by the double line can be scaled by either of the three pertinent transitional lengthscales (i.e., $\ell_{m *}$ is given by either $\ell_{m k}, \ell_{m \widetilde{m}}$, or $\left.\ell_{m \widetilde{o}}\right)$ since the coefficient $\beta_{-4}(\chi, \zeta)$ in front of it can be found only from the complete numerical solution. As a result, this term can be interpreted as correction in either toughness, leak-off, or leak-in. The corresponding expressions for the coefficient $\left(\beta_{-4}\right)_{*}$ are linked by the following relations: $\left(\beta_{-4}\right)_{k}=\chi^{4-6 h}\left(\beta_{-4}\right)_{\widetilde{m}}=\zeta^{4-6 h}\left(\beta_{-4}\right)_{\widetilde{o}}$.

The structure of the derived $m$-vertex expansion differes from that in the case of the Carter's leak-off (Garagash et al. 2011) by a single term, underlined in the above by a dotted line and corresponding to the pressure-dependent, leak-in correction, scaled by the $\ell_{m \widetilde{o}}=\left(E^{\prime} / \sigma_{o}^{\prime}\right)^{2}\left(\mu^{\prime} Q^{\prime} V^{1} / 2\right)^{2 / 3}$ transitional lengthscale.

\subsubsection{Expansion near $\widetilde{m}$-vertex}

The $\tilde{m}$-vertex solution may arise at intermediate distances $\max \left(\ell_{\widetilde{m} k}, \ell_{\widetilde{m} \widetilde{o}}\right) \ll x \ll \ell_{\widetilde{m} m}$ from the fracture tip, when the featured transitional lengthscales separate, within one of the corresponding limiting solution trajectories given by $k \rightarrow \widetilde{m} \rightarrow m,(3.5), \widetilde{o} \rightarrow \widetilde{m} \rightarrow m$, (3.9), or the combination thereof $k \rightarrow \widetilde{o} \rightarrow \widetilde{m} \rightarrow m$, (3.10), and shown by red and green colour in figure 2 . The essential condition for the existence of the intermediate $\widetilde{m}$ asymptote is therefore $\chi \gg 1$ and $\psi=\chi / \zeta \gg 1$, where the latter is always satisfied along the zero-leak-in (Carter's) edge, $\zeta=0$, previously explored by Garagash et al. (2011).

The asymptotic expansion about the $\widetilde{m}$-vertex solution, including small corrections due to toughness, storage, and pressure-dependent leak-off effects (see Appendix $\mathrm{C}$ for 
details) is given by

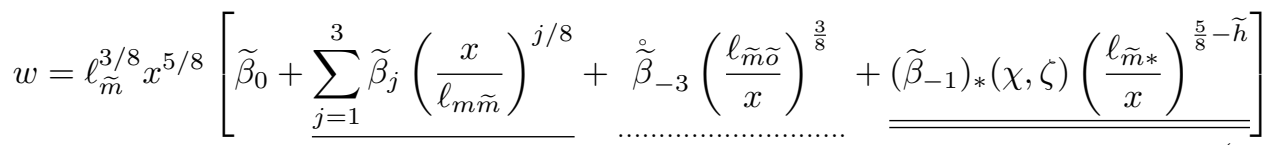

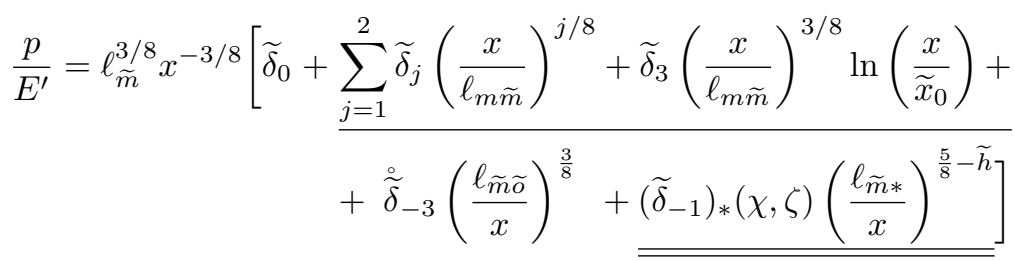

where $\widetilde{h}=0.0699928$ and known coefficients are given by $\widetilde{\beta_{0}}=2.53356, \widetilde{\beta}_{1}=$ $1.30165, \widetilde{\beta}_{2}=-0.451609, \stackrel{\check{\beta}}{-3}_{-0.524805}$, and by $\widetilde{\delta}_{j}=\widetilde{\beta}_{j} f\left(\frac{5}{8}+\frac{j}{8}\right)$ for $j=0,1,2$; $\stackrel{\widetilde{\delta}}{-3}_{-3}=\stackrel{\check{\beta}}{-3}_{-3} f(1 / 4) ; \widetilde{\delta}_{-1}=\widetilde{\beta}_{-1} f(\widetilde{h}) ;$ and $\widetilde{\delta}_{3}=\widetilde{\beta}_{3} / 4 \pi$. Parameters $\widetilde{\beta}_{-1}$ and $\widetilde{x}_{0}$ are a priori not known and are a part of the general numerical solution.

The terms underlined by the dotted and double lines correspond to the leak-in and toughness/leak-in corrections to the leading order ( $\widetilde{m}$-vertex) term within the $\tilde{m} \widetilde{o} k$ face solution, appropriately scaled with that face transitional lengthscales. The single line designates corrections due to fracture storage effects in the $m \widetilde{m}$ edge solution, appropriately scaled by that edge transitional lengthscale. In the zero storage case $\left(\ell_{\widetilde{m} m}=\infty\right)$, the leading term and terms underlined by the double line yield the far-field $\left(x \gg \ell_{\widetilde{m} k}\right)$ of the $\widetilde{m} k$-edge solution. Further, in the zero toughness case $\left(\ell_{\widetilde{m} k}=0\right)$, the leading term and terms underlined by the single line compose the near-field $\left(x \ll \ell_{\widetilde{m} m}\right)$ of the $\widetilde{m} m$-edge solution.

\section{Solution}

In this section, we will first introduce normalised governing equations based on different "edge" scalings (Table 3), followed by exploration of solutions in the problem parametric space (Figure 2): (i) the parameterless edge-solutions, (ii) one-parametric solution families of solutions for the faces of the parametric pyramid, and (iii) representive examples of two-parametric solution trajectories within the pyramid.

\subsection{Normalised equations}

Upon introducing normalised coordinate $\xi=x / \ell_{*}$, fracture opening $\Omega=w / w_{*}$ and net pressure $\Pi=p / p_{*}$, the corresponding normalised governing equations in different edge-scalings (table 3 ) are given in table 4 . Normalised equations are parameterised by a pair of the non-dimensional numbers; which, depending on the used scaling, are either $(\chi, \zeta)$ or $(\psi, \zeta)$, as defined in $(3.3),(3.6)$ and $(3.8)$.

When presenting the overall solution, we will make the most use of the $m k$-scaling, as it is based on the transition between the near $k$ and the far $m$ field behaviour of the general solution. In the limiting cases, when either one of the dissipation mechanisms or one of the storage mechanisms is negligible, corresponding to the four different (oneparametric) faces of the parametric pyramid $m \tilde{m} \widetilde{o} k$, we will use the scaling pertinent to the corresponding near-to-far transition. For example, the zero-leak-in face $m \widetilde{m} k\left(Q^{\prime}=0\right)$ and the zero-leak-off face $m \widetilde{o} k\left(C^{\prime} \propto \sigma_{o}^{\prime}=0\right)$, are both conveniently solved in the $m k$ - 
scaling with $\zeta=0$ (parametrised by $\chi$ ) and with $\chi=0$ (parametrised by $\zeta$ ), respectively. The zero-storage face $\widetilde{m} \widetilde{o} k\left(C^{\prime}=\infty\right)$, is conveniently solved in the $\widetilde{m} k$-scaling with $\chi=\infty$ (parametrised by $\zeta$ ). Finally, for the zero-toughness face $m \tilde{m} \widetilde{o}\left(K^{\prime}=0\right)$ and since the $m \widetilde{o}$-edge solution does not exist, we will use the $\widetilde{m} \widetilde{o}$-scaling with $\zeta=\infty$ (parameterised by $\psi=\chi / \zeta)$.

To compute the numerical solution, we utilise the numerical algorithm first developed by Garagash et al. (2011) which details are recounted in Appendix D.

\subsection{Edge solutions}

Fracture opening and net fluid pressure profiles for $m k, \widetilde{m} k, \widetilde{m} m$ edge solutions are shown in figure 3 and for the $\widetilde{m} \widetilde{o}$-edge in figure 4 , in their respective scalings. The former were previously obtained by Garagash et al. (2011) and are reproduced in figure 3 for completeness and validation of the used numerical algorithm. For the $\widetilde{m} \widetilde{o}$-edge solution, we estimate $B_{\widetilde{o}} \approx 3.322 \cdot w_{\widetilde{m} \widetilde{o}} / \ell_{\widetilde{m} \widetilde{o}}^{3 / 2}$ for the dimensional coefficient $B_{\widetilde{o}}$ of the $\widetilde{o}$-vertex $\left(w=B_{\widetilde{o}} x^{3 / 2}\right)$ realised in the near-field of this edge. As expected, the edge solutions correspond to the transition with distance from the tip between the corresponding pair of the vertex solutions, as indicated in figures 3 and 4 .

\subsection{Face solutions}

One-parametric families of solutions for the crack opening and net-pressure corresponding to the four limiting faces of the parametric pyramid (figure 2) are shown in figures 5-9 in their preferred scalings, (a), and in the form normalised by the respective far-field asymptote, (b). In these plots, we also show the vertex solutions, as correspond to the near, far, and (where appropriate) intermediate fields of a given face solution.

The Carter's, zero-leak-in $(\zeta=0), m \widetilde{m} k$-face solution is shown on figure 5 for various values of the leak-off number $\chi$ from $\chi=0$ to $\chi=100$. The former corresponds to the storage $m k$-edge solution trajectory $(k \rightarrow m)$, while the latter closely approximates the two-edge $(\widetilde{m} k$ and $m \widetilde{m})$ solution trajectory marked by the emergence of the intermediate $\widetilde{m}$-vertex asymptote $(k \rightarrow \widetilde{m} \rightarrow m)$. This face solution has been obtained previously by Garagash et al. (2011) and shown here for completeness.

The zero-leak-off $(\chi=0), m \widetilde{o} k$-face solution is shown on figure 6 in the $m k$-scaling for various values of the leak-in number $\zeta$ from $\zeta=0$ to $\zeta=20$. The former, once again, corresponds to the storage $m k$-edge solution, while the latter signals large leakin conditions. The solution is seen to evolve with increasing leak-in number such that the region dominated by the near-field $k$-asymptote expands outwards from the fracture tip, while the transition to the far-field $m$-asymptote takes place in increasingly abrupt fashion, as particularly evident for the crack opening (figure 6 left). The net pressure near field behaviour is dominated by the nearly constant (tip) value, which domain is seen to expand outward from the tip with increasing leak-in number. For large values of $\zeta$, the net pressure initially decreases with the distance from the tip (signalling the dominance of leak-in and the reversed direction of the fluid flow inside the crack channel there), passes through the minimum, and eventually recover towards the zero value, as solution transitions towards the far field $m$ asymptote. The net-pressure minimum becomes increasingly abrupt with increasing $\zeta$, marking effective pinching of the fracture there and spatially correlating with the maximum crack opening gradient. The crack is effectively closed over the enlarging with $\zeta$ region adjacent to the fracture tip, such that its effective tip corresponds to the 'pinching' at the net-pressure minimum. No emergent intermediate $\widetilde{o}$-vertex (3/2 opening slope) is evident with increasing $\zeta$ (which would have 
(a) $m k$-edge $(\chi=0, \zeta=0)$
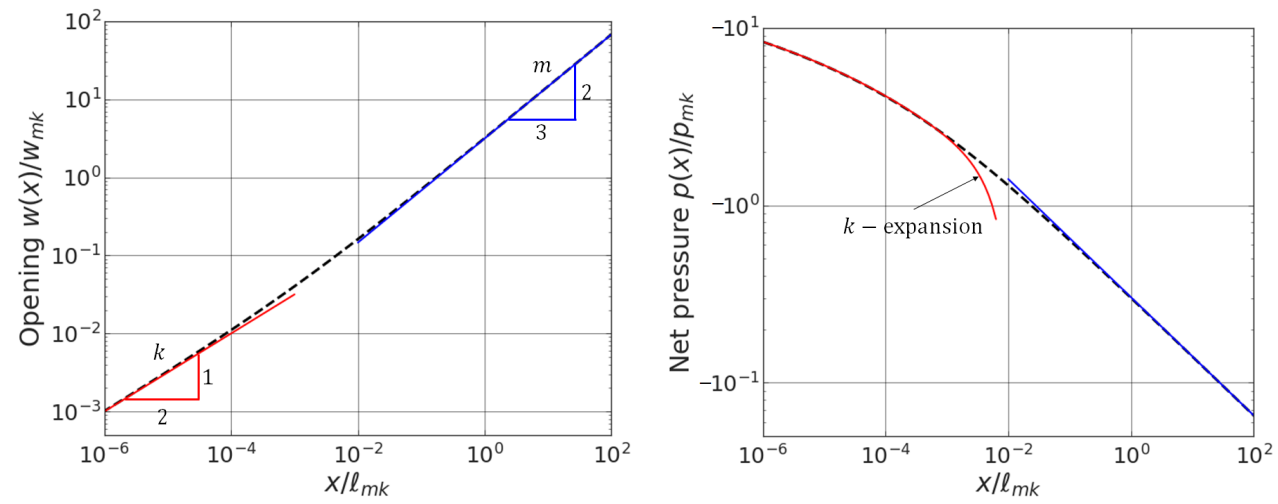

(b) $\widetilde{m} k$-edge $(\chi \rightarrow \infty, \zeta=0)$
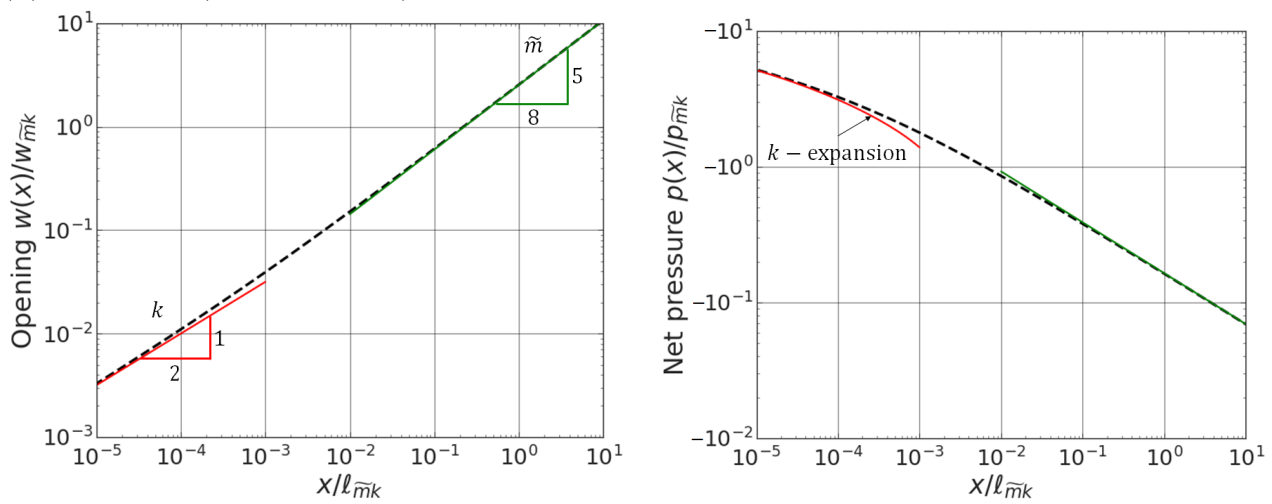

(c) $m \tilde{m}$-edge $(\chi \rightarrow \infty, \zeta=0)$
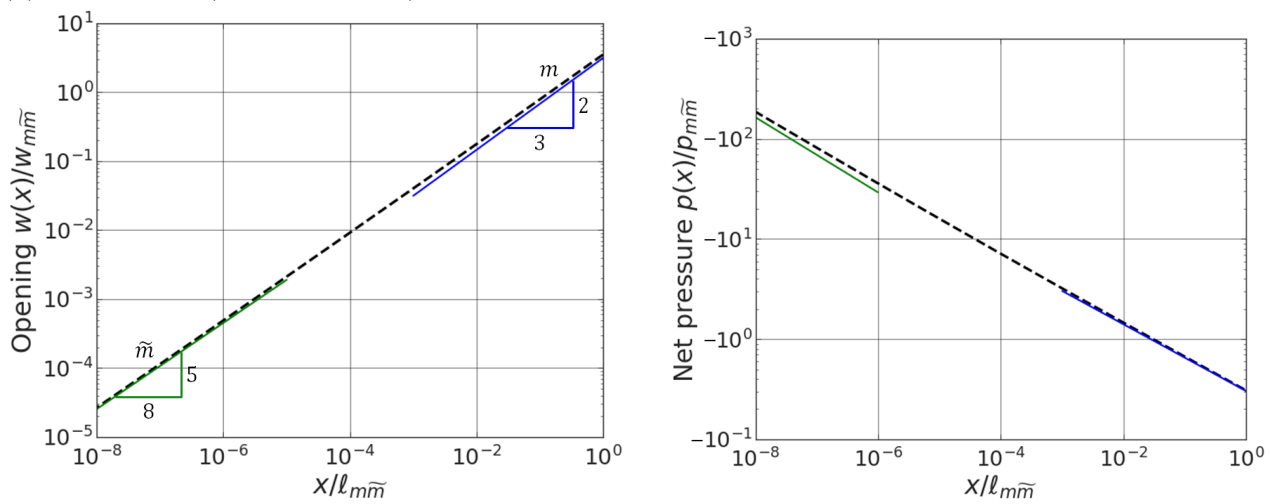

Figure 3: Fracture opening (left) and net fluid pressure (right) profiles with distance from the crack tip for edges: (a) $m k$, (b) $\widetilde{m} k$ and (c) $\widetilde{m} m$ in their pertinent scalings (Table 3 ). Various vertex solutions corresponding to the near and far field asymptotes are also shown.

led to the two-edge limiting solution trajectory $k \rightarrow \widetilde{o} \rightarrow m$ ), underscoring the previous assertion that the $m \widetilde{o}$-edge solution does not exist in the limit $\zeta \gg 1$ limit.

The zero-storage $(\chi=\infty), \widetilde{m} \widetilde{o}-$ face solution is shown on figure 7 in the $\widetilde{m} k$-scaling for various values of the leak-in number $\zeta$ from $\zeta=0$ to $\zeta=10$. As previously, the former 

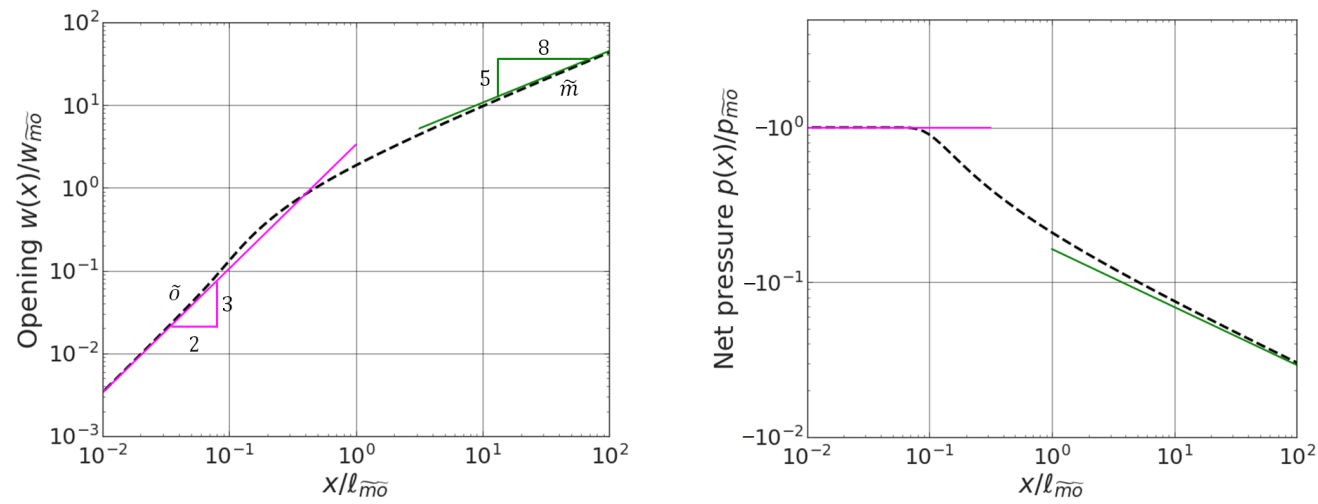

Figure 4: Fracture opening (left) and net fluid pressure (right) profiles with distance from the crack tip for the $\widetilde{m} \widetilde{o}$-edge in the pertinent scaling (Table 3 ). Vertex solutions corresponding to the near and far field asymptotes are also shown.

(a)
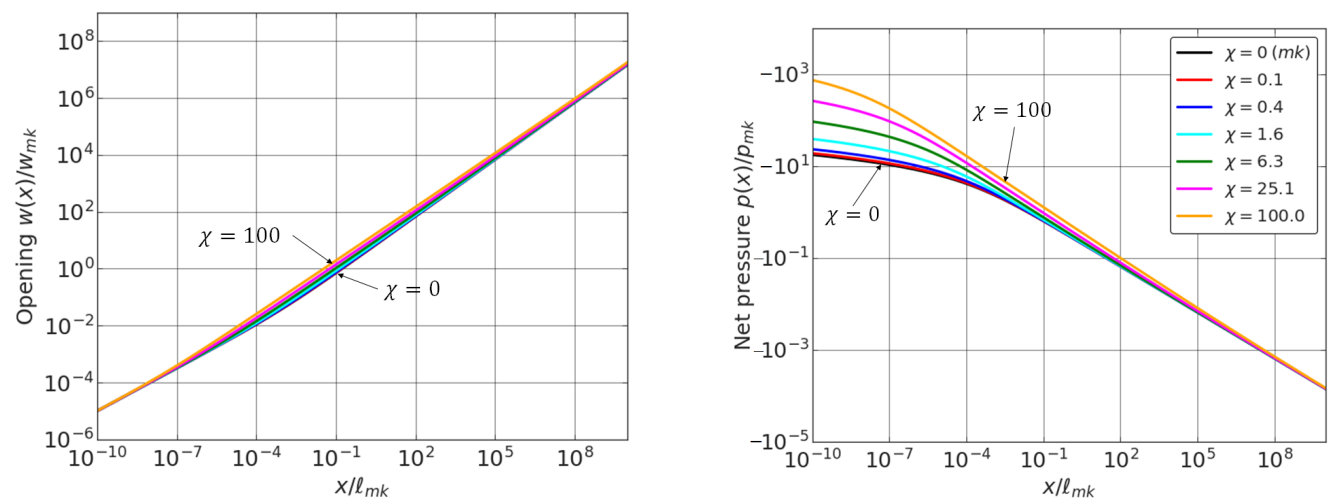

(b)
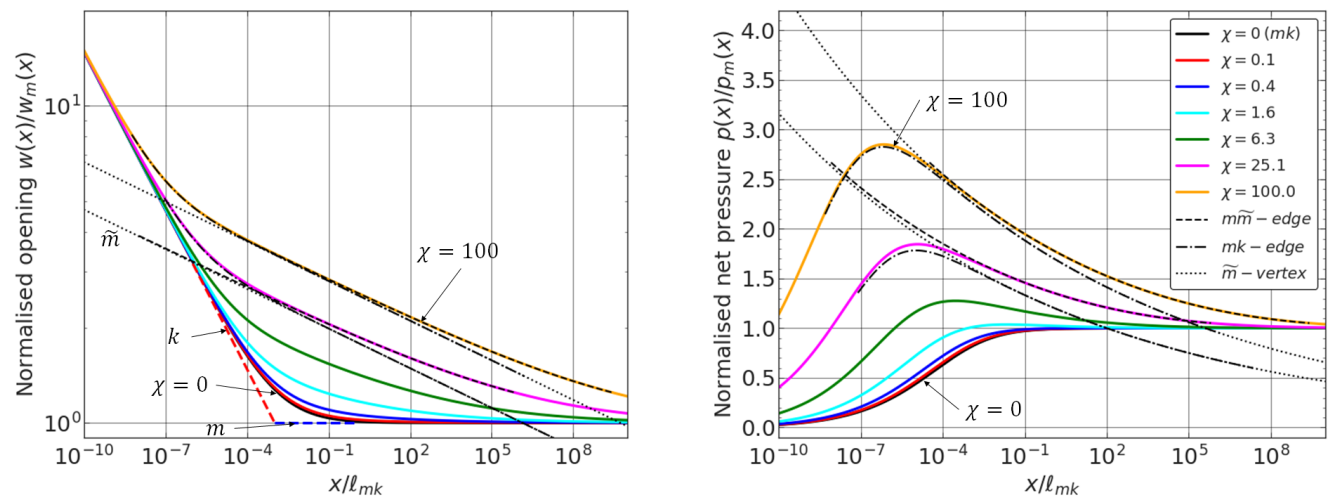

Figure 5: Zero-leak-in $(\zeta=0) m \tilde{m} k$-face solution for the fracture opening (left) and net fluid pressure (right) profiles in the $m k$-scaling for various values of the leak-in number $\zeta$. Solutions are shown in (a) the explicit form and (b) normalised by the $m$-vertex solution. The $\widetilde{m} k-, m \widetilde{m}$-edges, and $k, m$ and $\widetilde{m}$-vertices are also shown in (b). 
(a)
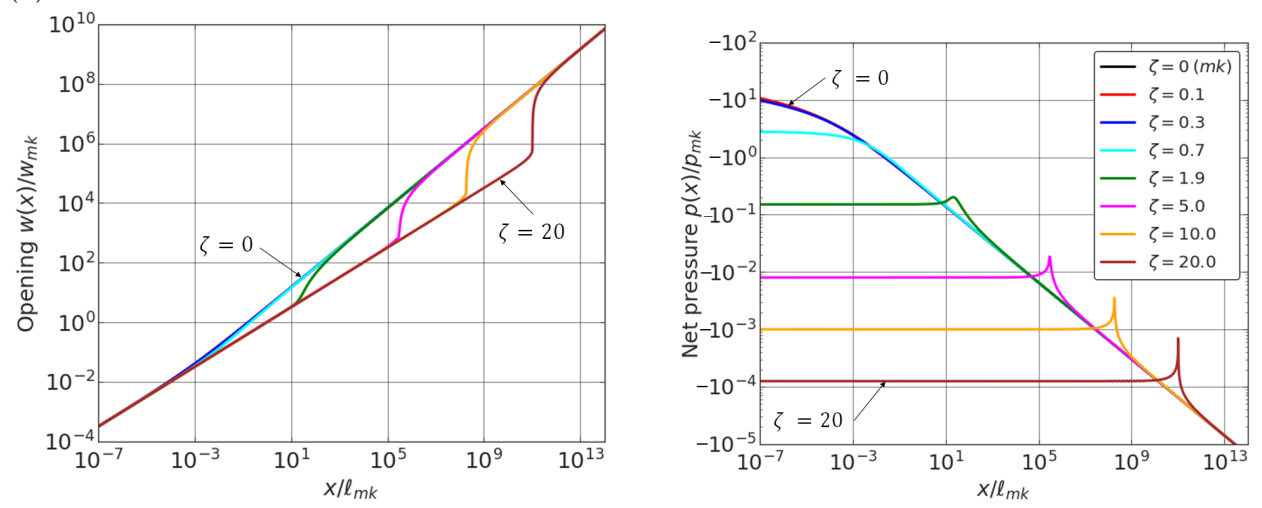

(b)
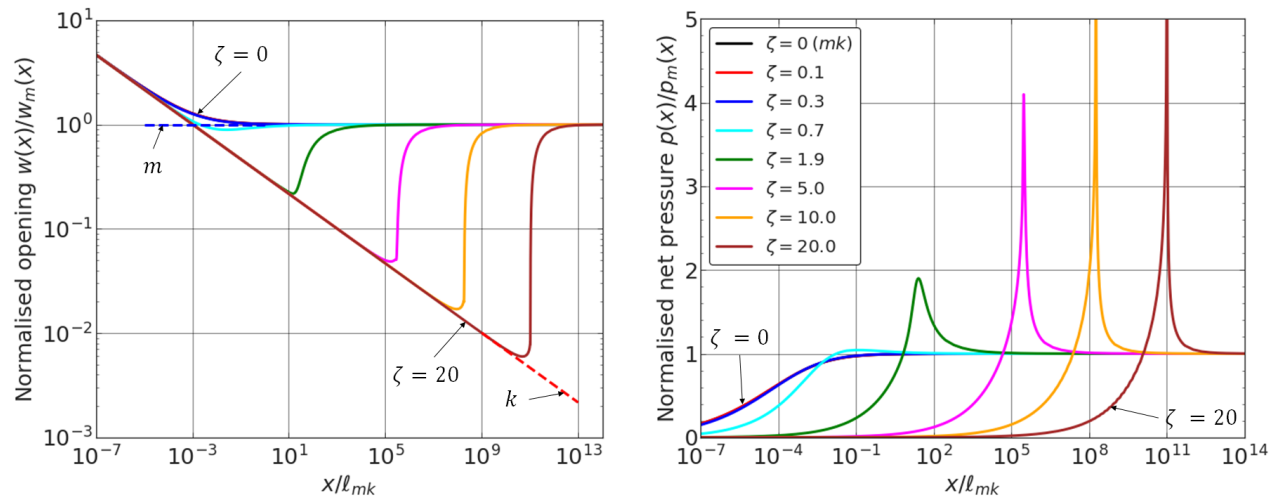

Figure 6: Zero-leak-off $(\chi=0) m \widetilde{o} k$-face solution for the fracture opening (left) and net fluid pressure (right) profiles in the $m k$-scaling for various values of the leak-in number $\zeta$ : (a) explicit form and (b) normalised by the $m$-vertex solution. The $k$ and $m$-vertices are also shown in (b).

corresponds to the $m k$-edge solution, while the increasing leak-in leads to somewhat similar evolution of the solution to that within the $m \widetilde{o} k$-face considered in the above (figure 6). I.e. increasing leak-in leads to the expansion of the near $k$ field outward from the crack tip, seen as the nearly constant net pressure (tip) value on figure $7 \mathrm{a}$ (left), with one important distinction from the $m \widetilde{o} k$-face in that the net-pressure is now monotonically increasing everywhere along the crack, without developing a pinching point (the local minimum). As a result, the intermediate $\widetilde{o}$ behaviour is seen to emerge at large leak-in $(\zeta=10)$, indicating the convergence of the solution trajectory towards the two-edge ( $\widetilde{o} k$ and $\widetilde{m} \widetilde{o}$ ) trajectory, $k \rightarrow \widetilde{o} \rightarrow \widetilde{m}$. This trend is explored for yet larger values of $\zeta$ in figure 8 .

The zero-toughness $(\chi=\infty), m \widetilde{m} \widetilde{o}$-face solution is shown on figure 9 for various values of the effective-stress number $\psi=\chi / \zeta$ from $\psi=0.5$ to $\psi=100$. The small $\psi$ value solution is approaching the non-existing $m \widetilde{o}$-edge limit, which is, as discussed previously in the context of approaching $m \widetilde{o}$-edge from within the $\widetilde{m} \widetilde{o} k$-face, characterised by the net pressure minimum and the crack pinching point. Large $\psi$ solution approaches the limit of the two-edge $(\widetilde{m} \widetilde{o}$ and $m \widetilde{m})$ solution trajectory $\widetilde{o} \rightarrow \widetilde{m} \rightarrow m$ which is realized 
(a)
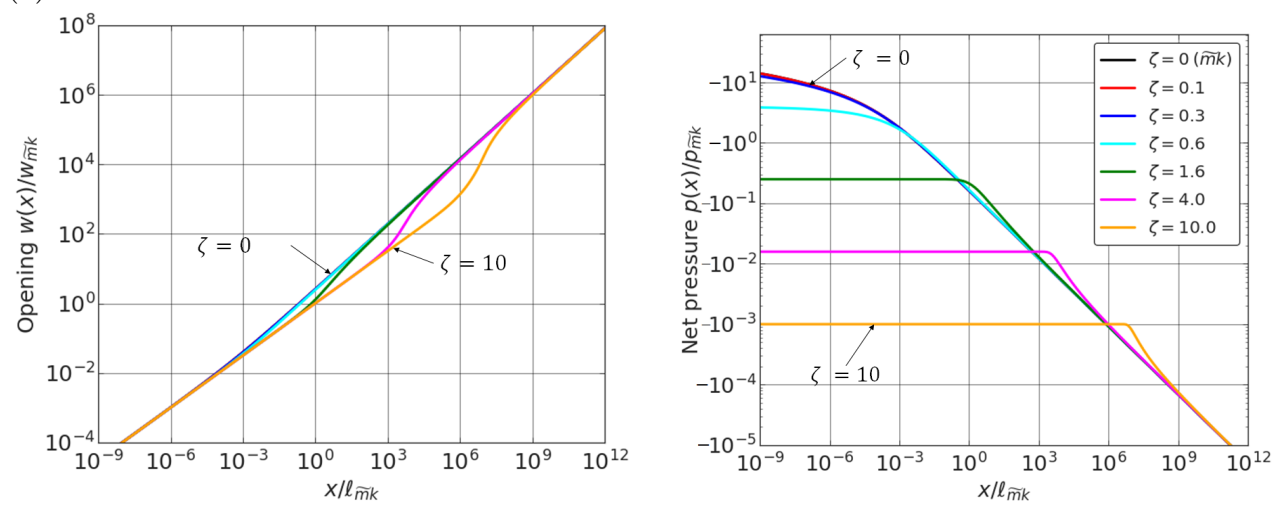

(b)
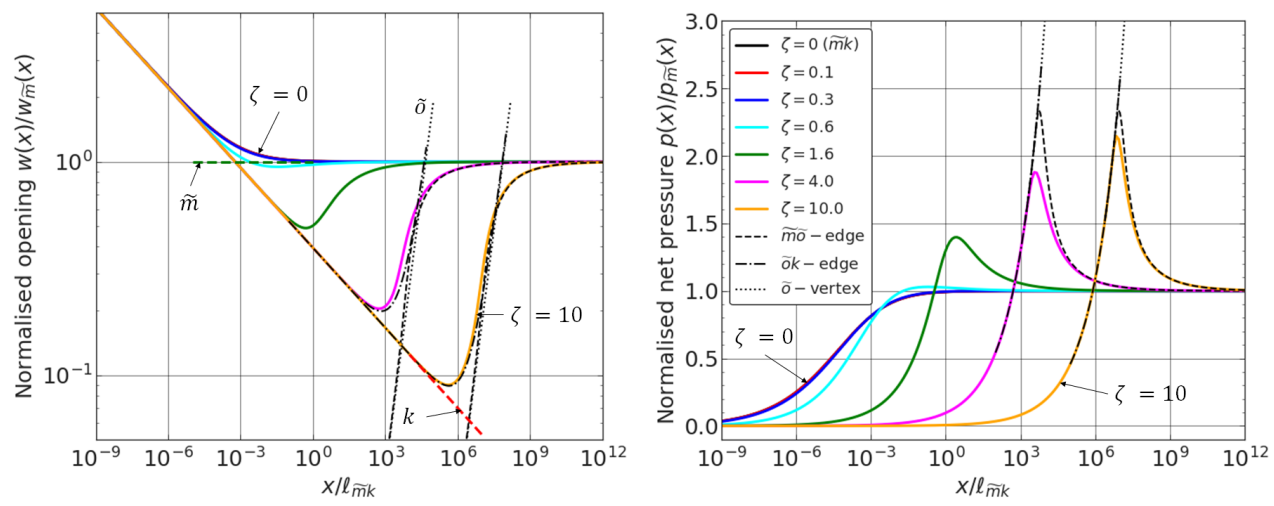

Figure 7: Zero-storage $(\chi=\infty) \widetilde{m} \widetilde{o}$-face solution for the fracture opening (left) and net fluid pressure (right) profiles in the $\widetilde{m} k$-scaling for various values of the leak-in number $\zeta$ : (a) explicit form and (b) normalised by the $\widetilde{m}$-vertex solution. The $\widetilde{m} \widetilde{o}-, \widetilde{o} k$-edges, and $k, \widetilde{m}$ and $\widetilde{o}$-vertices are also shown in (b).

over very wide range of distances from the tip (see figure 10 showing the solutions in the extended coordinate range).

\subsection{Examples of the general solution inside the parametric pyramid}

For presentation of particular solution trajectories within the parametric pyramid (i.e. when $0<\chi, \zeta<\infty)$ we choose several values of the leak-off parameter: $\chi=0.1,1$ and 10, and values of the leak-in parameter $\zeta$ are selected so that to maintain constant $O(1)$ non-dimensional leak-off-to-leak-in ratio $\chi / \zeta^{3}=1$, i.e. $\zeta=\chi^{1 / 3}=0.46,1,2.15$, respectively. Fracture opening and net fluid pressure profiles for the aforesaid cases are shown in the $m k$-scaling on figure 11 and normalised by the $m$-vertex solution on figure 12. The corresponding Carter's leak-off solutions $(\zeta=0)$ are also shown by dashed black lines for comparison. Additionally, in figure 12, we show the near-field $(k)$, the far-field $(m)$ and the intermediate-field $(\widetilde{m})$ vertex-solutions and their expansions (Section 3.3) by coloured dashed lines in order to underscore the corresponding asymptotic domains and degree of approximation for the solution afforded by the asymptotic expansions.

One of the distinguishing features of the obtained profiles as compared to the Carter's leak-off case is the finite value of the net fluid pressure at the fracture tip. In the 


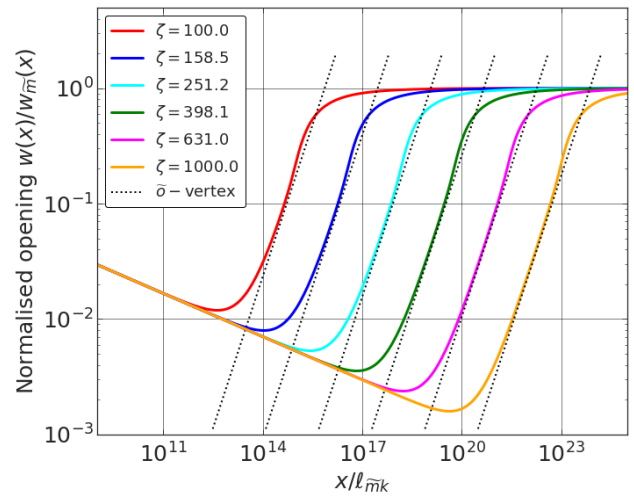

Figure 8: Zero-storage $(\chi=\infty) \widetilde{m} \widetilde{o}$-face solution for the fracture opening normalised by $\widetilde{m}$-vertex solution, as in figure 7 (left), but for larger values of leak-in number $\zeta$. The intermediate $\widetilde{o}$ asymptote is shown by dotted lines.

(a)
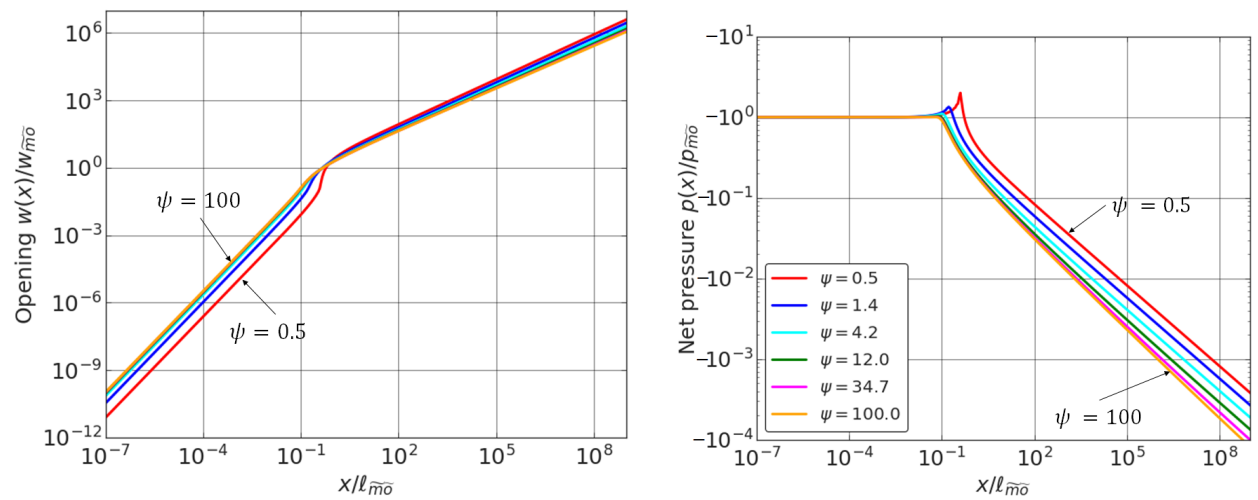

(b)
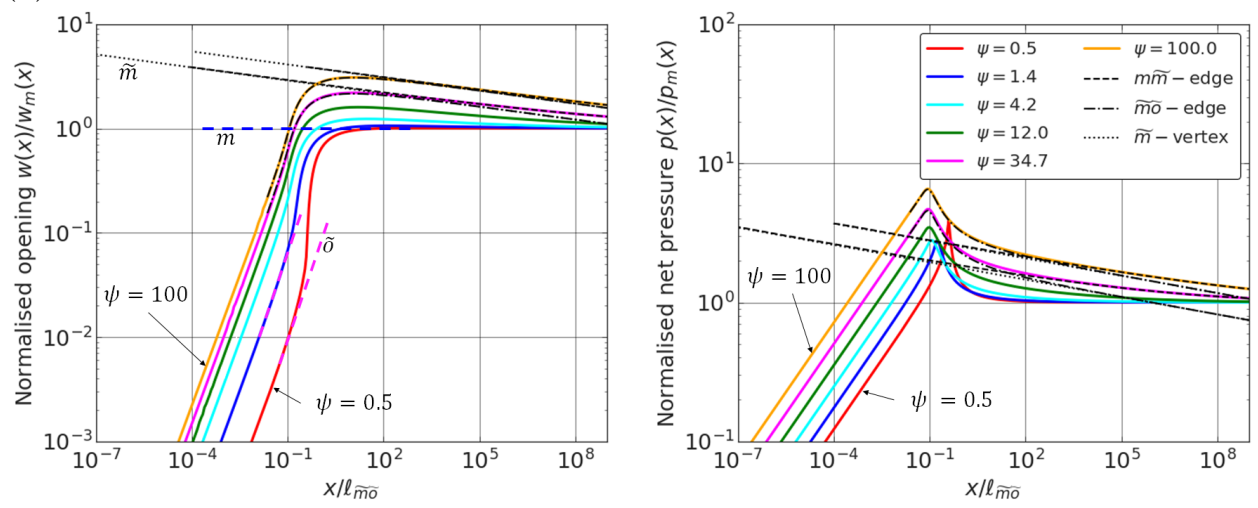

Figure 9: Zero-toughness $(\chi=\infty) m \widetilde{m} \widetilde{o}$-face solution for the fracture opening (left) and net fluid pressure (right) profiles in the $m \widetilde{o}-$ scaling for various values of the leak-off-toleak-in ratio $\psi=\chi / \zeta$ : (a) explicit form and (b) normalised by the $m$-vertex solution. The $\tilde{m} \widetilde{o}-, m \widetilde{m}-$ edges, and $\widetilde{o}, m$ and $\widetilde{m}$-vertices are also shown in (b). 

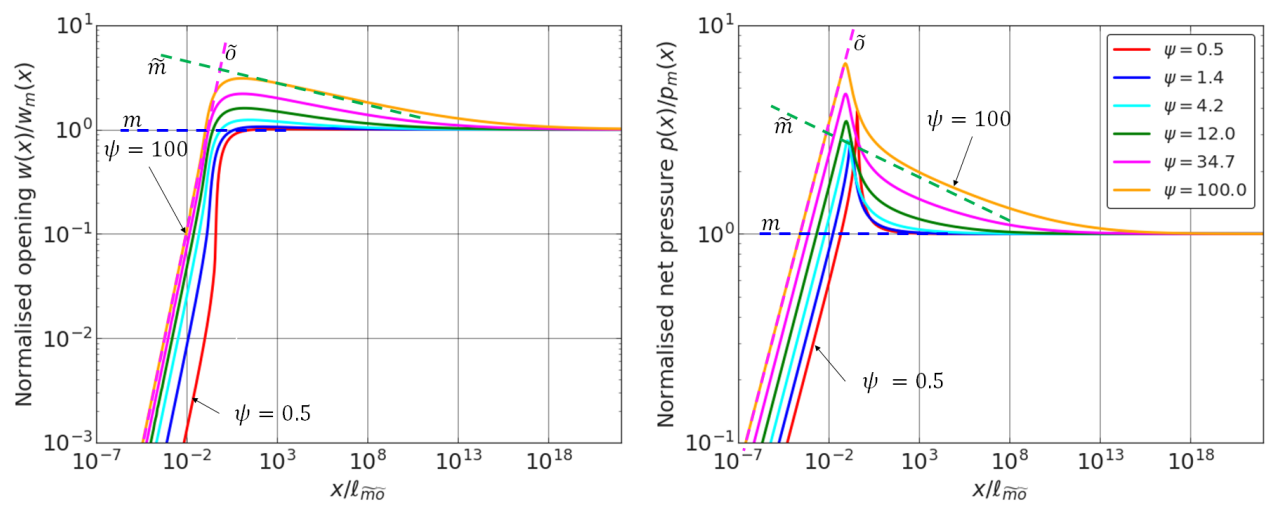

Figure 10: Zero-toughness $(\chi=\infty) m \tilde{m} \widetilde{o}$-face solution normalised by the $m$-vertex solution from figure $9 \mathrm{~b}$ shown in an extended coordinate range.

$m k$-scaling, it is defined by the equation: $p(0) / p_{m k}=-(\chi+1) / \zeta^{3}$ (table 2$)$. From figures 11a, 12a one could find out that the departure of the solution from the Carter's one is small for $\chi=0.1$ case, but it become more considerable for $\chi=1$ (figures $11 \mathrm{~b}$, $12 \mathrm{~b}$ ) and $\chi=10$ (figures 11c, 12c).

The applicability zone of the $m$-expansion shrinks when the value of the leak-off parameter $\chi$ increases. At the same time, the coordinate range, where $k$-expansion approximates the numerical solution, expands, and its length is much larger than in the Carter's leak-off case. Neither $\widetilde{m}$ nor $\widetilde{o}$-vertex solutions are realized as intermediate asymptotes in the solutions for the parametric choices in figures $11-12$, i.e. $\chi \leqslant 10$ and $\zeta \leqslant 2.15$, since the conditions for these intermediate behaviours call for $\chi, \psi=$ $\chi / \zeta \gg 1$ and $\chi, \zeta \gg 1$, respectively (see (3.9) and (3.7)). However, the intermediate $\widetilde{m}$-expansion (3.17), (3.18) does appear to closely approximate the numerical solution in the intermediate field in the case of $\chi=10$ (figure 12c) signalling the emergent intermediate asymptotic behaviour. Indeed, this trend persists in Figure 13, where we show the normalized solution for higher values of the leak-off and leak-in numbers, $\chi=100, \zeta=4.64$ and $\chi=1000, \zeta=10$. We observe that the solution is closely approximated by (i) the $\widetilde{m} \widetilde{o} k$-face solution $(\chi=\infty$, see figure 7$)$ and (ii) the $m \tilde{m}$-edge solution matched over intermediate distances from the tip. In other words, corresponding solution trajectories are approaching the limit of $k \rightarrow(\widetilde{o}) \rightarrow(\widetilde{m}) \rightarrow m$ (see green-coloured trajectory on figure 2) where parenthesised intermediate vertices are emergent within the considered solutions.

\section{Discussion}

\subsection{Representative values of problem parameters}

In order to frame the discussion of the obtained solutions to the hydraulic fracture tip problem, we consider estimates for typical values/ranges of dimensional problem parameters, as pertain to the application of hydraulic fracturing in petroleum reservoir stimulation field, and the corresponding ranges of the non-dimensional HF tip parameters $\chi$ and $\zeta$ (or their ratio $\chi / \zeta^{3}$ ). We base parametric estimates on two types of hydrocarbon reservoir rock: shale and sandstone, which typify the lower and higher limits of reservoir rock volumetric and filtration properties, respectively, while have similar geomechanical properties. Specifically, we take the following value ranges: 
(a) $\chi=0.1, \zeta=0.46$
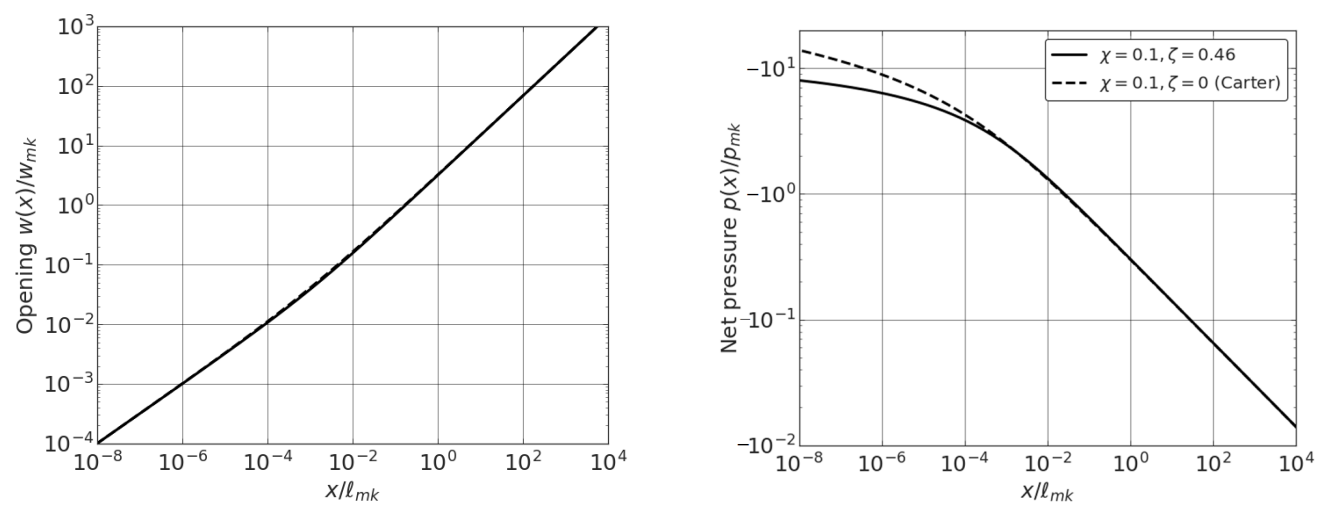

(b) $\chi=1, \zeta=1$
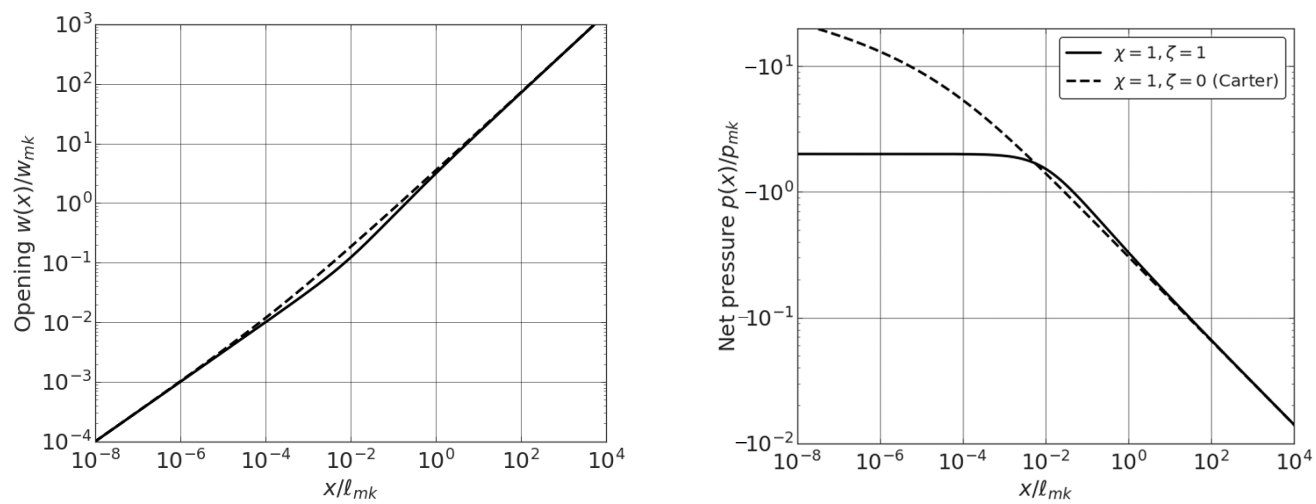

(c) $\chi=10, \zeta=2.15$
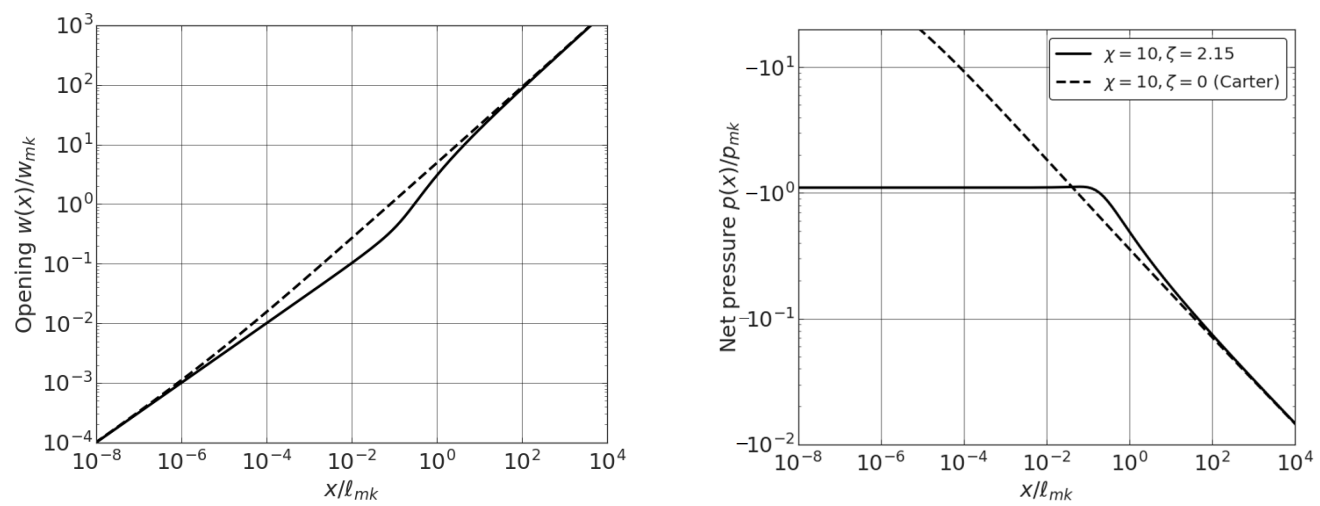

Figure 11: Solution for the fracture opening (left) and net fluid pressure (right) shown in the $m k$-scaling for fixed ratio $\chi / \zeta^{3}=1$ and different values of $\chi$ : (a) $\chi=0.1, \zeta=0.46$, (b) $\chi=1, \zeta=1$, (c) $\chi=10, \zeta=2.15$. The corresponding Carter's solutions $(\zeta=0)$ are shown by dashed lines for comparison. 
(a) $\chi=0.1, \zeta=0.46$
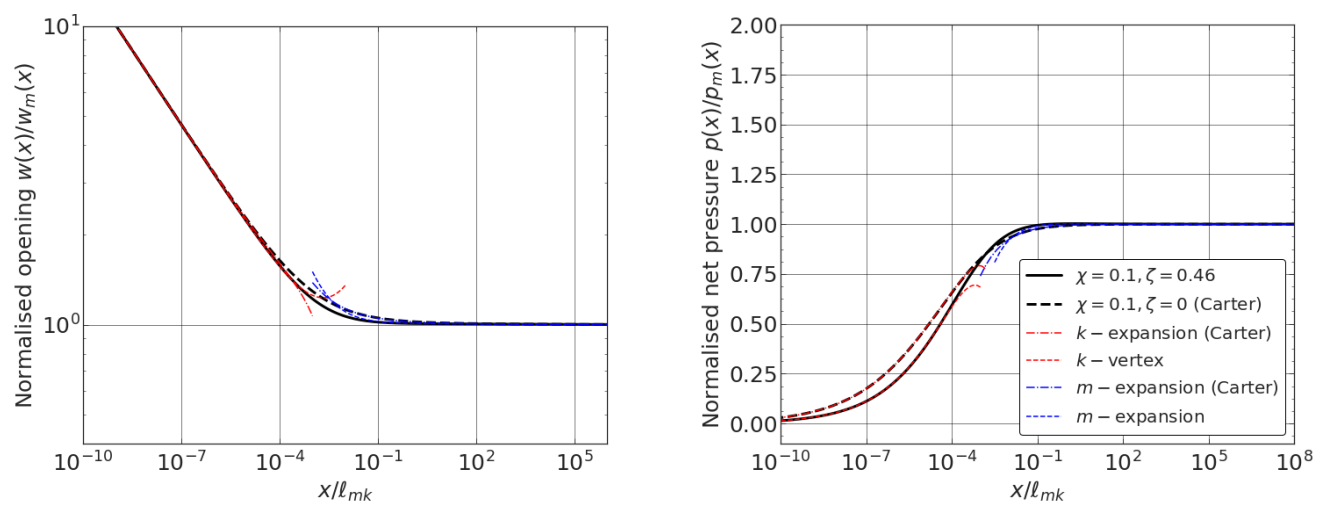

(b) $\chi=1, \zeta=1$
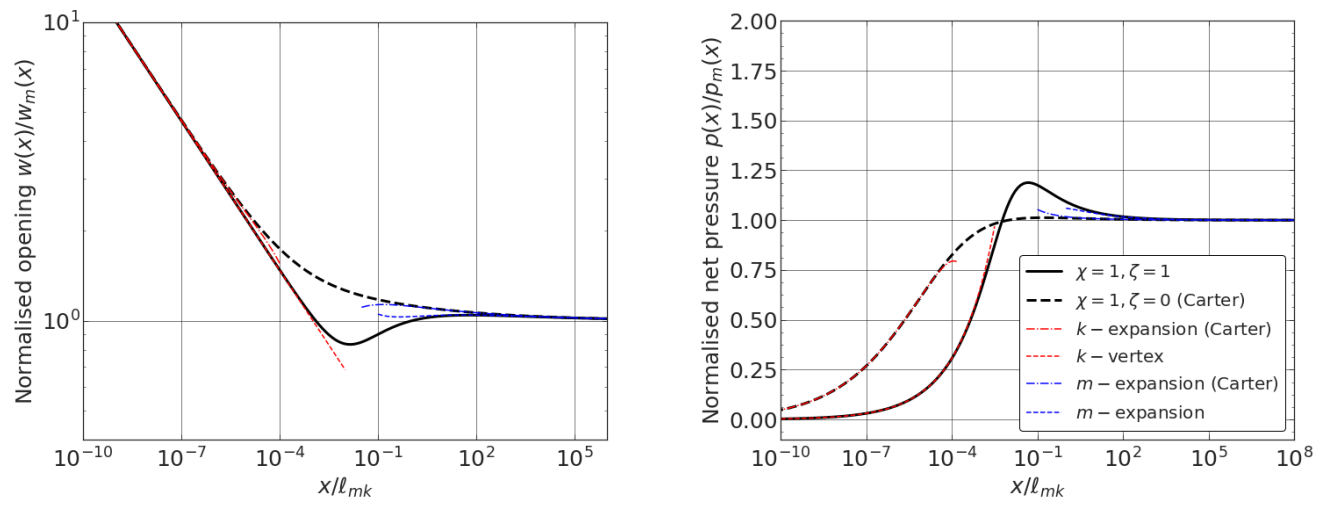

(c) $\chi=10, \zeta=2.15$
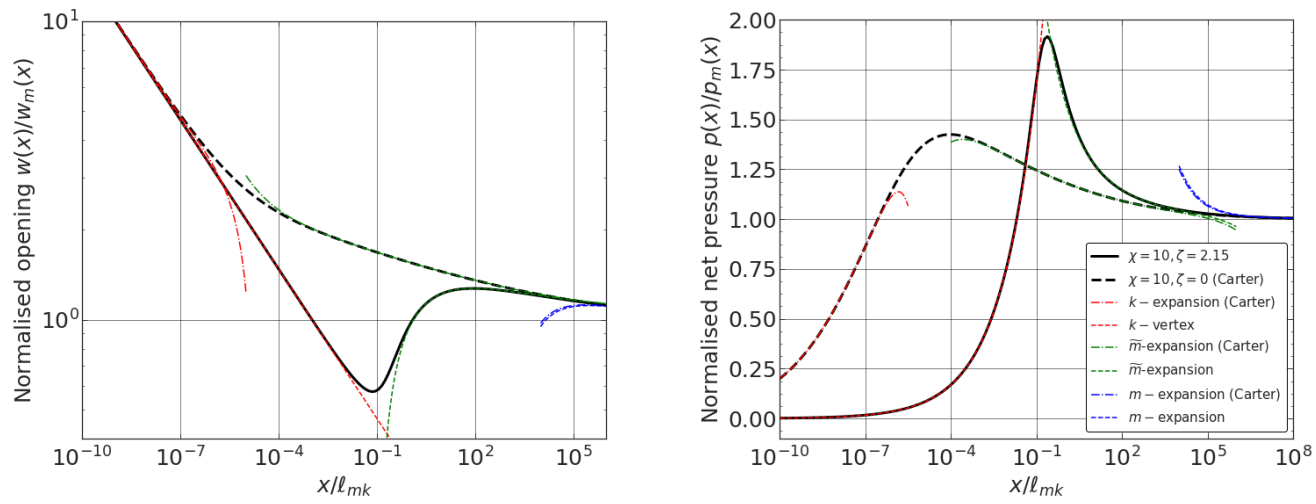

Figure 12: Solution for the fracture opening (left) and net fluid pressure (right) from figure 11 normalised by the far-field $m$-vertex solution. Near-, intermediate- $(\chi=10)$ and far-field asymptotic expansions are shown by dashed coloured lines.

- for the geomechanical properties and stress - plane-strain elastic modulus $E^{\prime}=30$ $\mathrm{GPa}$, pore volume total compressibility $c_{t}=1 / \mathrm{GPa}$ (water in pore space); rock fracture toughness $K_{I c}$ between 0.3 and $1 \mathrm{MPa} \cdot \sqrt{\mathrm{m}}$ (Chandler et al. 2016), and confining stress $\sigma_{o}=30 \mathrm{MPa}$ 

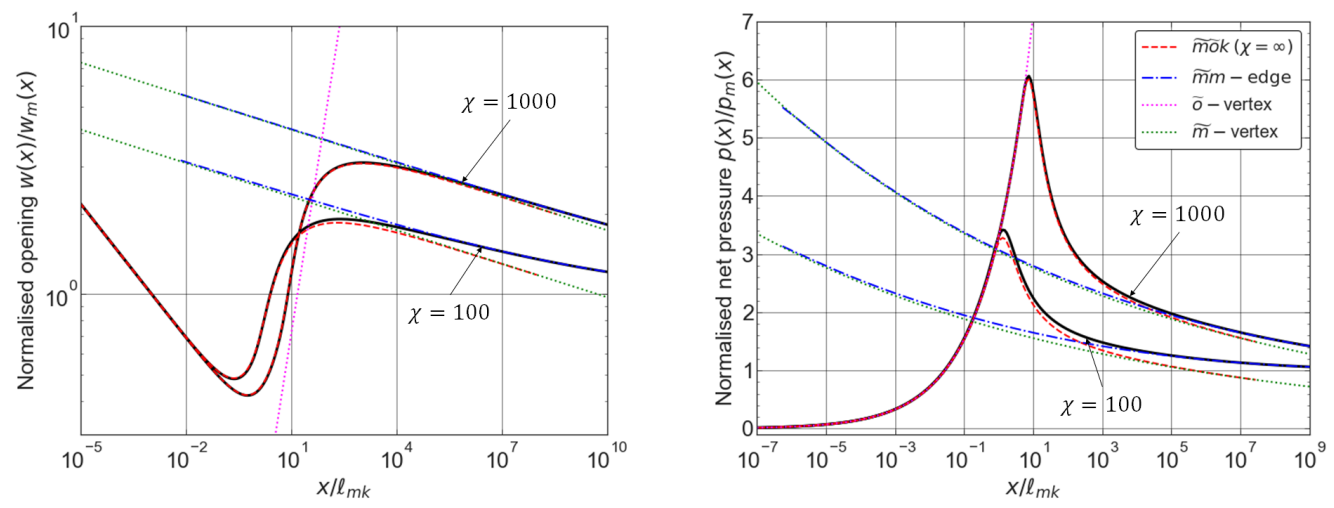

Figure 13: Fracture opening and net fluid pressure profiles normalised by the $m$-vertex solution for cases: $\chi=100, \zeta=4.64$ and $\chi=1000, \zeta=10$.

- for the formation reservoir properties and pore pressure - permeability in the range $k=0.1 \div 100 \mathrm{mD}$ (Li et al. 2016), porosity $\phi=5 \div 25 \%$ (Magara 1980; Manger 1963), and pore-pressure-to-stress-ratio $p_{o} / \sigma_{o}=0.95 \div 0.4$ (Walsh 1981) - where the bounds correspond to a shale and sandstone type reservoir (the latter is assumed to be at the hydrostatic pore pressure, while the former is overpressurized)

- fluid characteristics: $\mu=1 \mathrm{cP}$ (water), $5 \mathrm{cP}$ (slick water);

- fracture propagation velocity: $V$ between 0.1 and $1 \mathrm{~m} / \mathrm{s}$.

Figure 14 shows the parametric domain in the space of the HF tip non-dimensional parameters $\chi$ and $\chi / \zeta^{3}$ when the dimensional parameters are independently varied within the ranges described in the above (e.g., allowing for overpressured reservoirs with sandstone properties and normally pressured reservoirs with shale properties, etc). At each boundary of shown polygon one or two dimensional parameter are varied while the other remain fixed at their lower or upper bound (as applicable). The sense of change of $\left(\chi, \chi / \zeta^{3}\right)$ with increase of a given dimensional parameter are shown by arrows.

In addition we consider four specific limiting parametric choices corresponding to the overpressured-shale / normally-pressured-sandstone reservoir types (with parameteric values given by the lower/upper bounds of the assumed ranges), water / slick-water fluid types, while taking $K_{I c}=1 \mathrm{MPa} \cdot \sqrt{\mathrm{m}}$ and $V=1 \mathrm{~m} / \mathrm{s}$. The corresponding four parametric points $\left(\chi, \zeta, \chi / \zeta^{3}\right)$ are recorded in table 5 and shown by symbols on the map of figure 14 .

\subsection{Asymptotic fields and fluid-exchange domains}

We consider now boundaries of applicability of various asymptotic fields (vertex solutions) within the general HF tip solution. An asymptotic bound is defined here as a distance from the fracture tip where the crack opening solution deviates from the considered asymptote (e.g., $k, m$, etc. vertex) by $5 \%$. Specifically, we refer to $x_{0}$ as the upper boundary of the $k$-vertex asymptotic region $0<x<x_{0}$, and $x_{\infty}$ as the lower boundary of the $m$-vertex asymptotic region $x_{\infty}<x<\infty$. Similarly, we define the $5 \%$ asymptotic thresholds corresponding to the intermediate $\widetilde{m}, \widetilde{x}_{\infty}<x<\widetilde{x}_{0}$, and $\widetilde{o}$, $\widetilde{x}_{\infty}^{o}<x<\widetilde{x}_{0}^{o}$, asymptotes, whenever either of them is realised in the solution.

Furthermore, in order to characterise the effect of fluid-exchange on the hydraulic fracture tip solution, we introduce the boundary $x_{S}$ of the "crack-storage-domain" $x_{S}<$ $x<\infty$ where the rate of the cumulative fluid exchange between the fracture and the rock $q_{\perp}$ is a small fraction (less than $5 \%$ ) of the crack storage $w V, q_{\perp}\left(x_{S}\right)=0.05 \cdot w\left(x_{S}\right) V$.

In the complimentary domain $0<x<x_{S}$, the fluid exchange is non-negligible, and the 


\begin{tabular}{ccccc}
\hline & \multicolumn{2}{c}{ Sandstone } & \multicolumn{2}{c}{ Shale } \\
\hline HF tip parameter & Water & Slick water & Water & Slick water \\
\hline$\chi$ & 61 & 27.3 & 0.07 & 0.03 \\
$\zeta$ & 1.8 & 4 & 0.03 & 0.06 \\
$\chi / \zeta^{3}$ & 17 & 3.4 & 1.4 & 0.3 \\
\hline$\lambda / \ell_{m k}$ & $6 \cdot 10^{-5}$ & 0.01 & 0.02 & 2.2 \\
$x_{C} / \ell_{m k}$ & 0.5 & 32 & 174 & $2 \cdot 10^{4}$ \\
\hline
\end{tabular}

Table 5: Values of non-dimensional leak-off $\chi$, leak-in $\zeta$ parameters, and of ratio $\chi / \zeta^{3}$ for sandstone/shale reservoir, and water/slick-water fluid, as specified in the text. The corresponding solutions for the size of the near tip pore fluid circulation zone $\lambda$ and the boundary $x_{C}$ of the Carter's leak-off domain $\left(x>x_{C}\right)$ are also shown normalized by the $m k$ transitional lengthscale $\left(\ell_{m k} \approx 9 \mathrm{~m}\right.$ for water and $0.4 \mathrm{~m}$ for slick-water).

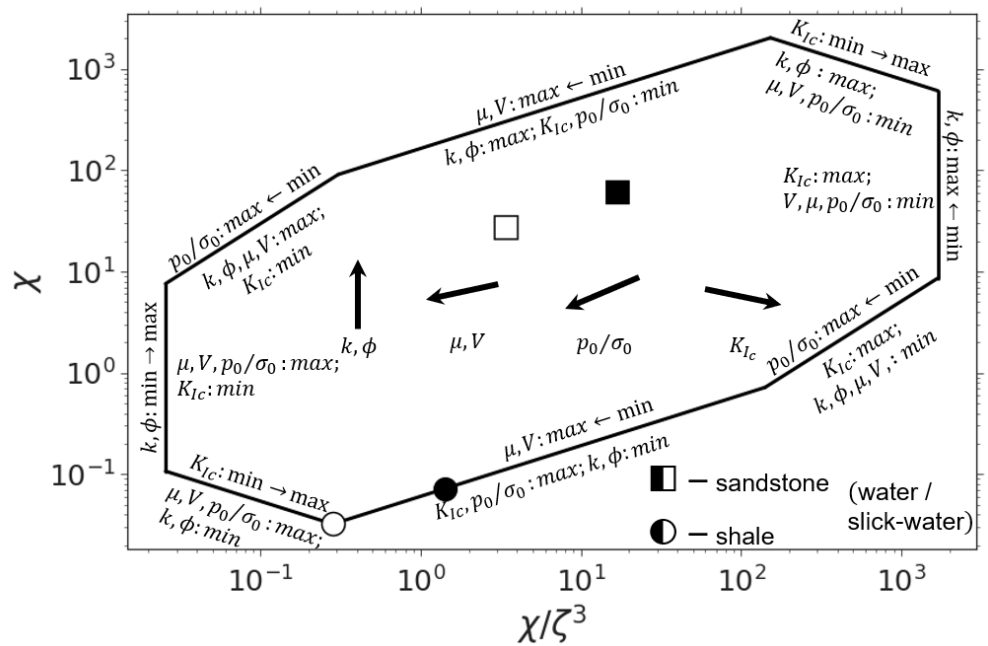

Figure 14: Parametric domain in terms of the non-dimensional leak-off number $\chi$ and leak-off-to-leak-in ration $\chi / \zeta^{3}$ representative of the field range estimates of the problem parameters. Symbols show particular field cases (sandstone vs. shale rock and slick-water vs. water fracturing fluid) from Table 5 .

relative significance of the Carter's leak-off and leak-in correction terms in the expression for $q_{\perp}$ can gauged by their ratio as a function of the distance from the tip,

$$
\operatorname{PDI}(x)=-\frac{1}{\sigma_{o}^{\prime} \sqrt{x}} \int_{0}^{x} \frac{p(s)}{2 \sqrt{x-s}} d s,
$$

which we refer to as the pressure-dependent leak-off index (PDI). We can use this function to evaluate the extent $\lambda$ of the near tip pore fluid circulation zone in the fracture, $0<$ 
$x<\lambda$, (figure 1), for which

$$
\operatorname{PDI}(\lambda)=1,
$$

and the boundary $x_{C}$ of the far field fracture domain dominated by Carter's leak-off, $x_{C}<x<\infty$, defined as distance from the tip where the cumulative leak-in correction is at $5 \%$ of the cumulative Carter's leak-off, i.e.

$$
\operatorname{PDI}\left(x_{C}\right)=0.05
$$

Figure 15 show maps of the asymptotic fields (left) and the various fluid-exchange domains (right) along the fracture $\left(x / \ell_{m k}\right)$ as a function of the leak-off number $\chi$ for four fixed values of the leak-off-to-leak-in ratio $\chi / \zeta^{3}=0.1,1,10$, and 1000 (a-d). For each of the above four cases, the corresponding range of $\chi$ representative of the field conditions (see parametric domain on figure 14) is indicated by a thick-line interval on the $\chi$-axis.

When considering the asymptotic vertex domains within the general solution (figure 15 (left)), we observe that the near-field $k$ domain expands with increasing leakin (corresponding to the decreasing leak-off-to-leak-in ratio $\chi / \zeta^{3}$ from the case with $\chi / \zeta^{3}=1000,(\mathrm{~d})$, to $\left.\chi / \zeta^{3}=0.1,(\mathrm{a})\right)$ over most of the shown leak-off $\chi$ range (vertical axis in figure 15). In turn, the far-field $m$ domain dependence on $\chi / \zeta^{3}$ is non-monotonic, as it is seen to expand from the case with $\chi / \zeta^{3}=10,(\mathrm{c})$, to $\chi / \zeta^{3}=1$, (b), and then shrink to the case with $\chi / \zeta^{3}=0.1$, (a). The former expansion of the $m$ and $k$ domains with diminishing $\chi / \zeta^{3}$ is likely linked to diminishing leak-off effects and the disappearance of the intermediate Carter's leak-off $\widetilde{m}$ behaviour, while the further $m$-domain contraction may be caused by proliferation of the pressure-dependent leakin effects at the smallest value of the ratio considered here $\chi / \zeta^{3}=0.1$, figure 15 (a). (This can be further substantiated by expanding size $\lambda$ of the pore-fluid circulation zone with diminishing $\chi / \zeta^{3}$, as seen on figure 15 (right)). The intermediate field $\widetilde{m}$-domain appears only in the case $\chi / \zeta^{3}=1000,(\mathrm{~d})$, when the pressure dependent leak-in effects are small, and, additionally, the leak-off is large $(\chi>50)$. The intermediate viscosity-leak-in $\widetilde{o}$ domain does not appear in all considered cases since the conditions of its existence $(\chi, \zeta \gg 1)$ are not met.

Let us now consider the effects of the fluid exchange between the fracture and the rock onto the solution summarised in the PDI maps on figure 15 (right). We observe that the crack-storage-dominated domain in the semi-infinite fracture $\left(x>x_{S}\right)$ shrinks, or migrates further away from the tip, with both (i) increasing leak-off $\chi$ at fixed leak-offto-leak-in ratio $\chi / \zeta^{3}$ and (ii) increasing pressure-dependent leak-in effects corresponding to diminishing value of the ratio $\chi / \zeta^{3}$, from $\chi / \zeta^{3}=1000,(\mathrm{~d})$, to $\chi / \zeta^{3}=0.1$, (a). The increasing influence of the leak-in with diminishing value of $\chi / \zeta^{3}$ is also reflected by overall increase of the pressure-dependence-index PDI (hotter colours in figure 15 (right)), corresponding growth of the near tip circulation zone $\lambda$, and shrinkage, or migration away from the tip, of the Carter's leak-off domain $\left(x>x_{C}\right)$.

The importance of the pressure-dependent fluid exchange effects to the propagation of a finite hydraulic fracture (for which we have provided the near tip solution here) can be gauged by comparing the Carter's leak-off boundary $x_{C}$ in the near tip solution with the representative lengthscale $\ell$ of the finite fracture, e.g., the radius of a penny-shape hydraulic fracture or the half-length of a plane-strain KGD fracture, etc. If $x_{C} \ll \ell$, then the pressure-dependent fluid exchange effects are not important on the scale of the parent finite fracture, as they are confined to the very small near tip region effectively shielded by the Carter's leak-off domain from the rest of the fracture. In this case, the Carter's leak-off model is an appropriate approximation. Otherwise, i.e. when $x_{C}$ comparable 
or larger than $\ell$, the pressure-dependent fluid exchange effects are prominent in the finite fracture propagation, and Carter's model should be abandoned. Since, the Carter's bound $x_{C}=\ell_{m k} \xi_{C}(\chi, \zeta)$ is a dynamic lengthscale (i.e. it depends on the fracture tip propagation velocity $V=d \ell / d t$ via the tip lengthscale $\ell_{m k}$ and via the non-dimensional tip parameters $\chi$ and $\zeta$, see corresponding definitions in table 2 and equations (3.3) and (3.6)), the regime of the fluid exchange (pressure-dependent vs. Carter's), as it corresponds to the ratio $x_{C} / \ell$ may change during the propagation.

To underscore the above discussion, consider a particular example of the HF propagation with $\chi=\zeta=1$, which, according to the parametric estimates in figure 14 and table 5, corresponds to a slick-water $\mathrm{HF}$ in a reservoir with intermediate values of hydraulic properties (roughly geometric mean of the 'shale' and 'sandstone' cases in table $5)$. Figure $15 \mathrm{~b}$ indicates that $\lambda \approx \ell_{m k}$ and $x_{C} \approx 3 \times 10^{3} \ell_{m k}$, while lengthscale $\ell_{m k}$ is in the range from 0.4 to 40 meters for the range of the propagation velocity $V$ from 1 to $0.1 \mathrm{~m} / \mathrm{s}$, respectively, $\left(K_{I c}=1 \mathrm{MPa} \times \mathrm{m}^{1 / 2}\right.$ and $\left.E^{\prime}=30 \mathrm{GPa}\right)$. Thus, in this case, the tip circulation cavity is of metric size, while the effects of the pressure-dependent fluid exchange can be neglected for fractures exceeding 10s kilometers in lengths (i.e. when $\ell>x_{C}$ ). If we are now to consider the limiting cases of the 'shale' and 'sandstone' reservoirs from table 5 , we observe that similar conclusions about the general inadequacy of the Carter's approximation (which calls for unrealistically long fracture) to slickwater fracture apply. However, in the 'shale' case the fracture tend to propagate in the storage-dominated regime $(\chi<0.1)$, which allows to reasonably neglect fluid exchange all together.

\subsection{Some limitations of the model}

\subsubsection{D pore pressure diffusion}

Our model of the pressure dependent leak-off is hinged on the assumption of the 1D pore pressure diffusion in the permeable rock surrounding the fracture. As pointed out by Detournay and Garagash (2003), the 1D assumption is approximately valid when the pore pressure perturbation introduced by fracturing is contained to a boundary layer abating the fracture that is thin compared to the characteristic lengthscale of fluid pressure change along the part of the fracture where the fluid exchange process is important. Taking for the latter the size $\lambda$ of the near-tip pore fluid circulation zone, and for the former the corresponding thickness of the pore pressure boundary layer $\sqrt{c t}$ built up over the time $t=\lambda / V$ it takes for the fracture tip to propagate distance $\lambda$, the $1 \mathrm{D}$ condition reads

$$
\lambda \gg \ell_{d}, \quad \ell_{d}=c / V
$$

Detournay and Garagash (2003) refer to this condition as the 'large velocity limit' of the circulation cavity problem in reference to the inverse dependence of 'diffusion lengthscale' $\ell_{d}$ on the fracture propagation velocity.

When evaluating the above $1 \mathrm{D}$ condition, it is convinient to express diffusion lengthscale $\ell_{d}$ in the $m k$-scaling, $\ell_{d} / \ell_{m k}=c E^{\prime 4} \mu^{\prime 2} V / K^{\prime 6}=(\pi / 16)\left(S E^{\prime}\right)^{-2} \zeta^{6}$. In the latter, the non-dimensional product $E^{\prime} S$ of the rock elastic modulus $E^{\prime}$ and rock pore space storativity $S=\phi c_{t}$ is weakely dependent on the rock type and can be estimated based on the previously discussed typical values of these parameters as $E^{\prime} S \sim 3$, resulting in $\ell_{d} / \ell_{m k} \sim 0.01 \zeta^{6}$. This estimate is shown in the maps in figures 15 (right) where it can be directly compared to the circulation zone length $\lambda / \ell_{m k}$. We observe that $1 \mathrm{D}$ fluid-exchange condition is satisfied for all considered values of $\chi$ in the case $\chi / \zeta^{3}=0.1$, figure $15(\mathrm{a})$, for $\chi \lesssim 0.1$ in the case $\chi / \zeta^{3}=1$, figure $15(\mathrm{~b})$, and finally, for for $\chi \lesssim 5$ in the case $\chi / \zeta^{3}=10$, figure $15(\mathrm{c})$. In other words, the $1 \mathrm{D}$ approximation of the pore pressure 
(a) $\chi / \zeta^{3}=0.1$
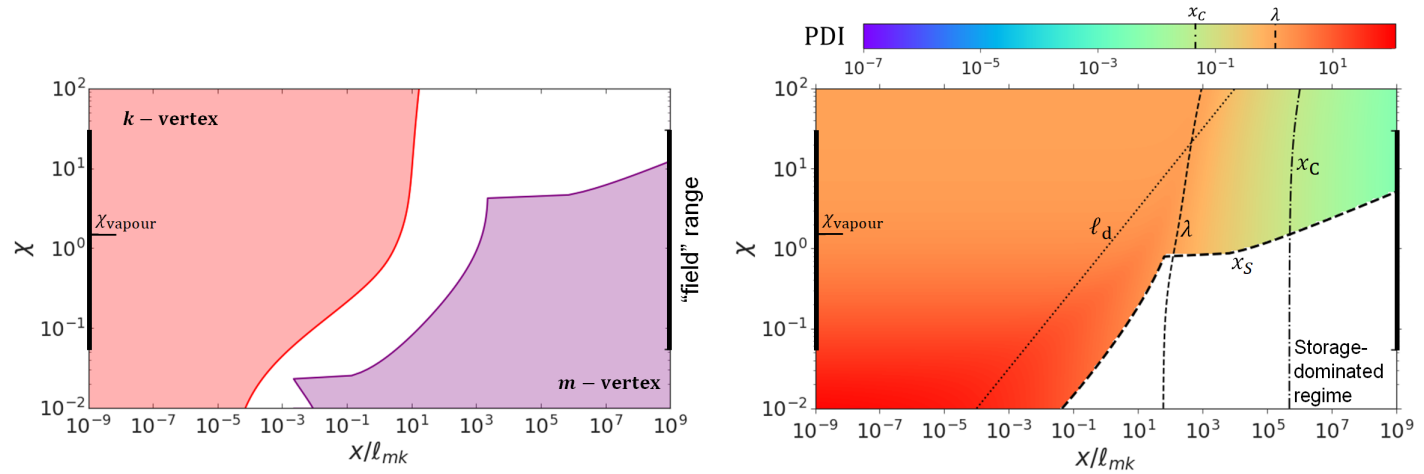

(b) $\chi / \zeta^{3}=1$
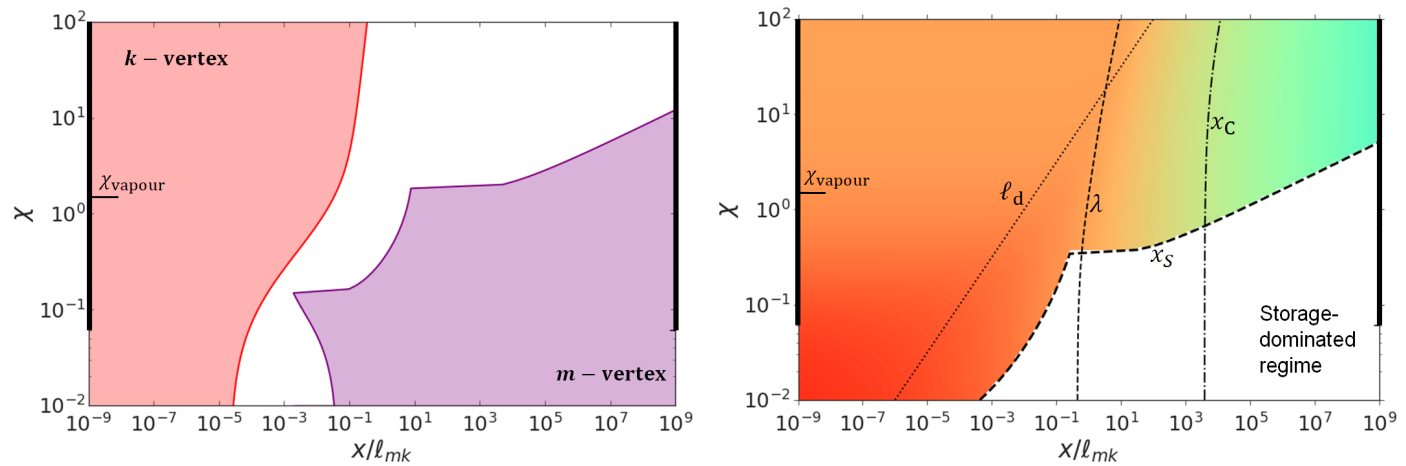

(c) $\chi / \zeta^{3}=10$
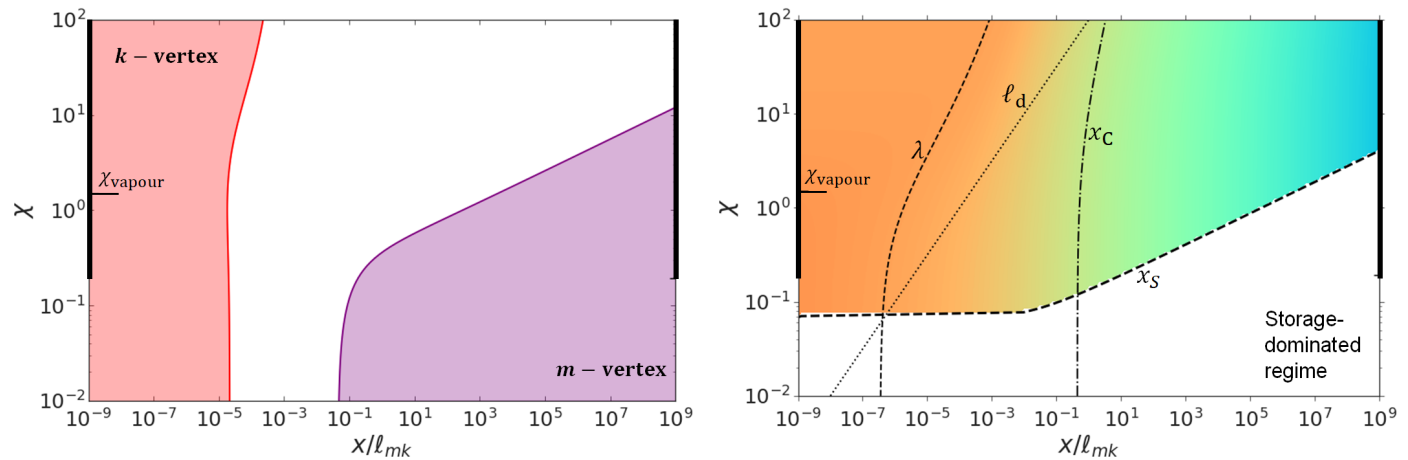

(d) $\chi / \zeta^{3}=1000$
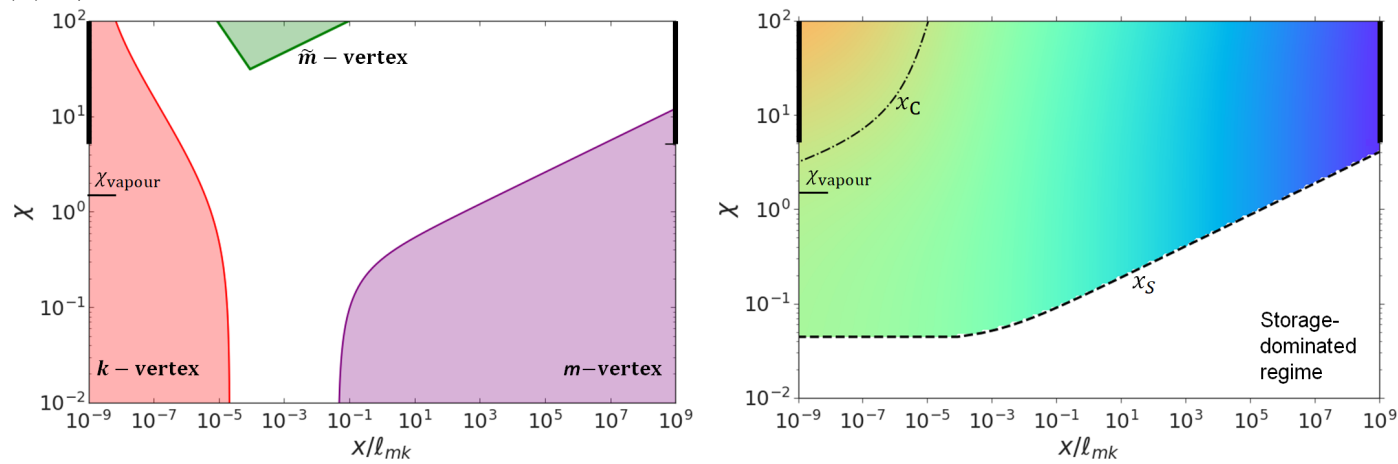

Figure 15: Regime maps (on the left) with applicability ranges of vertex solutions and PDI maps (on the right) with several characteristic boundaries $\left(\lambda, \ell_{d}, x_{C}, x_{S}\right)$ in four different cases: $\chi / \zeta^{3}=0.1$ (a), 1 (b), 10 (c), and 1000 (d). (Corresponding ranges of $\chi$ representative of the field (figure 14) are indicated by thick bar). 
diffusion is more readily justified when the pressure-dependent leak-in effects are more prominent (i.e. smaller values of the leak-off-to-leak-in ratio $\chi / \zeta^{3}$ and correspondingly larger circulation zone size $\lambda$ ).

\subsubsection{Sameness of the formation and fracturing fluids}

The assumption that the formation and fracturing fluids have identical properties is a restrictive one, in that it limits practical applications of this analysis (as the result, we focused here on the slick-water fracturing parametric examples, as the latter viscosity $\sim 5 \mathrm{cP}$ may be similar to that of the light oil). The future work will consider relaxing this assumption in order to extend the analysis to conventional fracturing fluids (polymeric gells) with the viscosity orders of magnitude larger than that of the formation fluid, and possibly to the 'cake building' due to leak-off (i.e. deposition of fracturing fluid solids and polymers into a thin, semi-solid, low-permeability 'cake' at the fracture wall). Kovalyshen and Detournay (2013) provide a workable theoretical framework to include these effects by distinguishing between the pore and fracturing fluid viscosities in the fluid flow in the fracture and tracking the 'cake' build-up, while reasonably assuming that the leaked-off filtrate (i.e. the base of the fracturing fluid when stripped from the solids and polymers) displacing the pore fluid in the permeable rock abating the fracture has properties identical to that of the formation pore fluid.

\subsubsection{Potential vaporisation of the pore fluid at the fracture tip}

The near tip region of the fracture dominated by the pore fluid leak-in corresponds to the absolute fluid pressure below the ambient field value $p_{o}$. Specifically at the tip, we have (table 2)

$$
p_{f}(0)=p_{o}-\Delta p_{\text {und }}, \quad \Delta p_{\text {und }}=\frac{K^{\prime} V^{1 / 2}}{E^{\prime} Q^{\prime}}=\frac{\sigma_{o}^{\prime}}{\chi}
$$

where $\Delta p_{\text {und }}$ corresponds to the undrained value of the pressure drop. The fluid will vapourize and form so-call 'fluid lag' adjacent to the fracture tip if pressure drops below the saturated vapour value. Taking the latter to be small compared to the reservoir ambient pore pressure value, the incipient fluid lag condition requires $p_{o}<\Delta p_{\text {und }}$, which in view of (5.3.3) can be rewritten in terms of the leak-off number

$$
\text { fluid lagging: } \quad \chi<\chi_{\text {vapour }}=\frac{\sigma_{o}^{\prime}}{p_{o}}=\frac{\sigma_{o}}{p_{o}}-1
$$

The above threshold value of $\chi$ can be estimated as $\chi_{\text {vapour }} \approx 1.5$ for normally-pressurised and $\approx 0.05$ for overpressured reservoirs. The normally-pressurized reservoir value of $\chi_{\text {vapour }}$ is indicated on the parametric maps of figure 15 . The vaporisation at the fracture tip, when predicted, does not necessarily invalidate the considered solutions, as long as the vapour-filled region (fluid lag) remains small compared to the predicted circulation zone size $\lambda$.

\section{Conclusions}

We analysed the near-tip region of a hydraulic fracture propagating in a permeable elastic solid, while allowing for pressure-dependent fluid exchange (leak-off and leakin) between the fracture and the host rock and associated pore pressure diffusion. In formulating the problem, we built on the original modelling framework of Detournay and Garagash (2003); Kovalyshen and Detournay (2013), which recognises the existence of the near fracture tip cavity dynamically filled by the pore fluid. The pore fluid is sucked from 
the rock into the dynamically depressurised fracture tip to be recirculated back into the rock some distance behind the tip. Asymptotic analysis of a number of limiting cases, including the reduction to the pressure-independent, Carter's leak-off case (Garagash et al. 2011), allowed us to frame the general structure of the solution and its parametric dependence within the space of two non-dimensional parameters dependent on the crack tip velocity $V$ : the leak-off $\chi \sim\left(E^{\prime} / K^{\prime}\right)\left(k \sigma_{o}^{\prime} / \mu \sqrt{c V}\right)$ and leak-in $\zeta \sim\left(E^{\prime} / K^{\prime}\right)(k \sqrt{V / c})^{1 / 3}$ numbers, respectively.

The full numerical solution of the near tip problem in $(\chi, \zeta)$ space provides a practical framework to understand the coupling of the physical processes near the fracture tip and its evolution with the crack tip velocity. The constructed maps of the near tip domains dominated by fracture toughness, fluid viscosity, and by pressure-dependent leak-off/leakin with distance from the tip (figure 15) allow to assess the propagation regime of a finite hydraulic fracture by contrasting the asymptotic domain boundaries to the length of the finite fracture (Garagash et al. 2011). For example, when considering representative field values of parameters for slick-water hydraulic fracturing, we found that the pressuredependent fluid-exchange domain in the near tip solution extends beyond the typical field fracture length, thus, invalidating the pressure-independent Carter's leak-off model in this case.

The obtained solution allows one to accurately model the interplay between the pore pressure and fluid pressure dynamics inside the fracture, captured by the pressuredependent leak-off (and leak-in), and their combined impact on the transient propagation of a finite hydraulic fracture, e.g., within the Planar3D approach (Peirce 2015; Dontsov and Peirce 2017). In doing so, the near tip solution obtained in this work may be numerically implemented into a module for the growth of a finite fracture in the form of a so-called tip element, used to match the fracture opening in the near-tip zone between the global numerical solution and the local near-tip asymptotics.

\section{Acknowledgements}

This work received financial support from the Ministry of Education and Science of the Russian Federation (project N 14.581.21.0027, unique identifier RFMEFI58117X0027). Startup funds of Skolkovo Institute of Science and Technology are gratefully acknowledged by A. A. Osiptsov.

\section{Appendix A. The rate of fluid exchange between the fracture and the ambient rock}

Under previously stated assumptions that (i) pore and fracturing fluid have the same properties and (ii) the characteristic distance over which fluid pressure varies along the fracture is much larger than the thickness of the diffusive boundary layer in the fracturenormal direction, the pore pressure diffusion at any fixed point $X$ on the fracture plane $(Y=0)$ can be approximated as one dimensional problem of diffusion into half-space $Y>0$ with ambient pore pressure $p_{r}(t=0)=p_{r}(Y \rightarrow \infty)=p_{o}$ from the prescribed source located on the fracture face. This source is characterised by fluid pressure inside the fracture which evolution in time can be prescribed by $p_{r}(X, Y=0, t)=p_{f}(x)$ where $x=V t-X$ is the time-dependent distance of the fixed point $X$ from the moving crack tip, and $p_{f}(x)$ is the 'stationary' fluid pressure profile in the steadily advancing fracture.

Solution to this problem can be furnished by time-convolution of the unit pressure step 
solution, or Green's function, (Carslaw and Jaeger 1959):

$$
p_{G}(Y, t)=\operatorname{erfc}(Y / \sqrt{4 c t})
$$

where $c=k / \mu S$ is the diffusivity coefficient in terms of the reservoir permeability $k$ storage coefficient $S$, and $\operatorname{erfc}(\cdot)$ is the complementary error function. Corresponding Green's function for the local rate of fluid exchange between the fracture and the rock follows by applying the Darcy's law:

$$
g_{G}(t)=-2 \frac{k}{\mu} \nabla p_{r \mid Y=0}=2 \frac{k}{\mu \sqrt{\pi c t}}
$$

where prefactor 2 accounts for the exchange across two identical fracture faces.

At $t=0$ when the fracture front first arrives at the considered point along the fracture plane, the fluid pressure undergoes a jump $p_{f}(0)-p_{o}$ from the ambient value $p_{o}$ in the rock to that $p_{f}(0)$ at the fracture tip. By applying convolution integral to the function $g_{G}(t)$, we obtain the dependency of fluid exchange rate on time:

$$
g(t)=\left(p_{f}(0)-p_{o}\right) g_{G}(t)+\int_{0}^{t} \frac{d p_{f}\left(V t^{\prime}-X\right)}{d t^{\prime}} g_{G}\left(t-t^{\prime}\right) d t^{\prime}
$$

This expression is further recorded in Eq. (2.5) in the main text after substituting for time in terms of the distance from the moving crack tip, $t=(x+X) / V$. Further integrating the local fluid exchange rate from the tip $x=0$ to some distance away from the tip $x>0$, we can obtain an expression for the local 'cumulative' fluid exchange rate $q_{\perp}(x)=$ $\int_{0}^{x} g\left(x^{\prime}\right) d x^{\prime}$. This expression, after some simplifications involving interchanging of the order of integration in the resulting double integral and integrating the corresponding transformed form, is recorded in Eq. (2.7) of the main text.

\section{Appendix B. Asymptotic expansion near $k$-vertex}

The leading term in the opening expansion for $x \rightarrow 0$ is given by the propagation condition $w=\ell_{k}^{1 / 2} x^{1 / 2}$ written in terms of the toughness lengthscale $\ell_{k}$. To obtain the corresponding leading term in the net pressure expansion, we write the fluid balance equation in terms of the viscosity $\ell_{m}$, leak-off $\ell_{\widetilde{m}}$, and new leak-in $\ell_{\widetilde{o}}=\left(\mu^{\prime} Q^{\prime} V^{1 / 2}\right)^{2 / 3}$ lengthscales as follows (similar to the treatment of Appendix B of (Garagash et al. 2011))

$$
w^{2} \frac{d p / E^{\prime}}{d x}=\ell_{m}+\ell_{\tilde{m}}^{3 / 2} \frac{x^{1 / 2}}{w}+\frac{\ell_{\widetilde{o}}^{3 / 2}}{w} \int_{0}^{x} \frac{p(s) / E^{\prime}}{2 \sqrt{x-s}} d s
$$

Further, taking the integral by parts on assumption that $p(0)<\infty$, we can write

$$
w^{2} \frac{d p / E^{\prime}}{d x}=\ell_{m}+\left(\ell_{\widetilde{m}}^{3 / 2}+\ell_{\widetilde{o}}^{3 / 2} \frac{p(0)}{E^{\prime}}\right) \frac{x^{1 / 2}}{w}+\frac{\ell_{\widetilde{o}}^{3 / 2}}{w} \int_{0}^{x} \frac{d p / E^{\prime}}{d s} \sqrt{x-s} d s
$$

Further, assuming that the pressure gradient at the tip is also bounded, and using the leading term expression for the opening, we observe that the left hand side $(\propto$ fluid velocity) and the integral term vanish when $x \rightarrow 0$, suggesting that to the leading order

$$
\frac{p(0)}{E^{\prime}}=-\frac{\ell_{\widetilde{m}}^{3 / 2}+\ell_{m} \ell_{k}^{1 / 2}}{\ell_{\widetilde{o}}^{3 / 2}}=-\sigma_{o}^{\prime}-\frac{K^{\prime} V^{1 / 2}}{E^{\prime} Q^{\prime}}
$$

as recorded in table 2 of the main text.

The next order (non-constant) term in the net pressure expansion corresponds to the 
following linear equation on the net pressure gradient, as results from (B 1) with (B 2) and use of the opening leading asymptote,

$$
x^{3 / 2} \frac{d p}{d x}=\left(\frac{\ell_{\widetilde{\sigma}}}{\ell_{k}}\right)^{3 / 2} \int_{0}^{x} \frac{d p}{d s} \sqrt{x-s} d s
$$

where the lengthscale ratio can be conviniently expressed in term of the leak-in nondimensional number $\zeta, \ell_{\widetilde{o}} / \ell_{k}=\zeta^{2}$. This linear equation possesses a power law solution which we choose to write in the form $d p / d x=-p(0) \zeta^{3}\left(x / x_{o}\right)^{\gamma(\zeta)-1}$ where $x_{o}$ is unknown constant and the exponent $\gamma(\zeta)$ is given implicitely by

$$
\frac{2}{\sqrt{\pi}} \frac{\Gamma\left(\gamma+\frac{3}{2}\right)}{\Gamma(\gamma)}=\zeta^{3}
$$

This prescribes monotonically increasing $\gamma(\zeta)$ from zero in the Carter's limit $\zeta \rightarrow 0$, $\gamma \sim \zeta^{3}$, to infinity when $\zeta \rightarrow \infty, \gamma \sim \pi^{1 / 3} \zeta^{2 / 3}$.

The corresponding $k$-vertex expansion of the net-pressure follows by integration

$$
\frac{p}{E^{\prime}}=\frac{p(0)}{E^{\prime}}\left[1-\frac{\zeta^{3}}{\gamma}\left(\frac{x}{x_{o}}\right)^{\gamma}\right]
$$

This form, upon substituting expression for $p(0)$ is given in Eq. (3.11) of the main text. The above net pressure expansion for $\zeta>0$ can be formally reduced to the expression given by (Garagash et al. 2011) in the Carter's limit $\zeta \rightarrow 0$, as given by Eq. (3.12) in the main text. Indeed, the latter follows upon noticing that, first, $p(0) / E^{\prime}=-\zeta^{-3} \ell_{k}^{1 / 2} / \ell_{1}^{1 / 2}$ where $\ell_{1}=\left(\ell_{m k}^{-1 / 2}+\ell_{\widetilde{m} k}^{-1 / 2}\right)^{-2}$ is the lengscale introduced by (Garagash et al. 2011) in the Carter's case, second, $\gamma \sim \zeta^{3}$ and $x^{\gamma} / \gamma \sim 1 / \gamma+\ln x$ when $\zeta \rightarrow 0$.

To obtain the next order term(s) in the $k$-vertex expansion for the opening, we follow the approach of (Garagash et al. 2011) (Appendix B) by evaluating the crack elasticity integral (2.3) using the net-pressure expansion (B 4), truncating the upper limit of integration to some finite value $X$, and then expanding the result for small $x \rightarrow 0$

$$
\begin{aligned}
w-\ell_{k}^{1 / 2} x^{1 / 2} & =\frac{4}{\pi} \int_{0}^{X} K(x, s) \frac{p(0)}{E^{\prime}}\left[1-\frac{\zeta^{3}}{\gamma}\left(\frac{s}{x_{o}}\right)^{\gamma}\right] d s \sim \\
& \sim-\zeta^{3} \frac{p(0)}{E^{\prime}}\left[\frac{x^{3 / 2}}{x_{1}^{1 / 2}}+\frac{4 \tan \pi \gamma}{\gamma(1+\gamma)} \frac{x^{\gamma+1}}{x_{o}^{\gamma}}\right]
\end{aligned}
$$

where contributions to the $x^{3 / 2}$-term from the both terms in the net pressure expansion are dependent on the truncated value of $X$, and thus, a priori unknown part of the full numerical solution for semi-infinite fracture, lumped in the above into a single unkwnon prefactor $x_{1}^{-1 / 2}$. As further discussed in the main text the order of the non-leading $x^{3 / 2}$ and $x^{\gamma+1}$ terms in the opening expansion depends on the value of $\zeta$, specifically, the former is dominant among the two when $3 / 2<\gamma(\zeta)+1$, which takes place when $\zeta>0.862$, and the oppositeis true, i.e. $x^{\gamma+1}$ is dominant among the two, when $\zeta<0.862$.

\section{Appendix C. Asymptotic expansion near $\widetilde{m}$-vertex}

The intermediate leak-off dominated asymptotic solution in the $m k$-scaling (Table 3 ) has the form: $\Omega(\xi)=\widetilde{\beta}_{0} \chi^{1 / 4} \xi^{5 / 8}, \Pi(\xi)=\widetilde{\delta}_{0} \chi^{1 / 4} \xi^{-3 / 8}$. This asymptotic behaviour arises in the distance range $\max \left(\ell_{\widetilde{m} k}, \ell_{\widetilde{m} \widetilde{o}}\right) \ll x \ell_{\widetilde{m} m}$ (in dimensional coordinate $x$ ). In the parametric space the essential condition for $\widetilde{m}$ asymptote existence is $\chi \gg 1$ and $\psi=\chi / \zeta \gg 1$ which is a consequence of transition lengthscales separation. 
Firstly, we introduce "bar" variables that is normalised values on the leak-off asymptote.

$$
\bar{\Omega}(\xi)=\frac{\Omega(\xi)}{\chi^{1 / 4} \xi^{5 / 8}}, \bar{\Pi}(\xi)=\frac{\Pi(\xi)}{\chi^{1 / 4} \xi^{-3 / 8}}
$$

In these new variables lubrication equation can be rewritten in the form:

$$
\xi \frac{d \bar{\Pi}}{d \xi}-\frac{3 \bar{\Pi}}{8}=\left(\frac{\xi}{\chi^{6}}\right)^{1 / 8} \frac{1}{\bar{\Omega}^{2}}+\frac{1}{\bar{\Omega}^{3}}+\frac{\zeta^{3}}{\bar{\Omega}^{3} \xi^{1 / 2} \chi^{3 / 4}} \int_{0}^{\xi} \frac{\bar{\Pi}\left(\xi^{\prime}\right) \xi^{\prime-3 / 8}}{2 \sqrt{\xi-\xi^{\prime}}} d \xi^{\prime}
$$

We find expansion terms using monomial solutions in the following form:

$$
\bar{w}_{\lambda}(\xi)=B \xi^{\lambda}, \bar{\Pi}_{\lambda}=B f\left(\frac{5}{8}+\lambda\right) \xi^{\lambda}
$$

where parameter $\lambda$ should satisfy the following condition $0<\frac{5}{8}+\lambda<1$

Let us firstly consider zero-storage case $\left(\ell_{\widetilde{m} m}=\infty\right)$. Here we should consider the farfield $\left(x \gg \max \left(\ell_{\widetilde{m} k}, \ell_{\widetilde{m} \tilde{o}}\right)\right.$ or $\left.\xi \gg \max \left(\chi^{-2}, \zeta^{8} \chi^{-2}\right)\right)$ of the $\widetilde{m} \widetilde{o} k$ pyramid face. In this limiting case the first term in the right-hand side of Eq. (C1) is absent:

$$
\xi \frac{d \bar{\Pi}}{d \xi}-\frac{3 \bar{\Pi}}{8}=\frac{1}{\bar{\Omega}^{3}}+\frac{\zeta^{3}}{\bar{\Omega}^{3} \xi^{1 / 2} \chi^{3 / 4}} \int_{0}^{\xi} \frac{\bar{\Pi}\left(\xi^{\prime}\right) \xi^{\prime-3 / 8}}{2 \sqrt{\xi-\xi^{\prime}}} d \xi^{\prime}
$$

Further, we identity the presence of the small parameter in the interested limit $(\xi \rightarrow \infty)$ that is in the pressure-dependent leak-off term. Since we consider $\bar{\Pi}(\xi)$ in the form of monomial solution: $\bar{\Pi}(\xi) \sim \xi^{\lambda}$, this term has the following form: $\sim \zeta^{3} \cdot \xi^{\lambda-3 / 8} \cdot \chi^{-3 / 4}$. According to the condition for $\lambda$ parameter, we know that this power is less than zero and, therefore, pressure-dependent term includes the small parameter $\left(\zeta^{8} /\left(\xi \chi^{2}\right)\right)^{3 / 8}$. So, we could represent the "bar" solution in the form of the summation of Taylor and non-Taylor terms:

$\bar{\Omega}(\xi)=\widetilde{\beta}_{0}+\left(\frac{\zeta^{8}}{\xi \chi^{2}}\right)^{3 / 8} \stackrel{\odot}{\beta}_{-3}+\frac{\widetilde{\beta}_{-1}(\chi, \zeta)}{\left(\chi^{2} \xi\right)^{\frac{5}{8}-\widetilde{h}}}, \bar{\Pi}(\xi)=\widetilde{\beta}_{0} f(5 / 8)+\left(\frac{\zeta^{8}}{\xi \chi^{2}}\right)^{3 / 8} \widetilde{\widetilde{\beta}}_{-3} f(1 / 4)+\frac{\widetilde{\beta}_{-1}(\chi, \zeta)}{\left(\chi^{2} \xi\right)^{\frac{5}{8}-\widetilde{h}}} f(\widetilde{h})$

Substituting this expansion into the lubrication equation for 'bar' functions in this particular limit, we could match coefficient in front of appropriate terms:

$$
\widetilde{\beta}_{0}=2.53356, \widetilde{\beta}_{-3}=-0.52481
$$

and for non-Taylor term we obtain the following equation for $\widetilde{h}$ :

$$
-\widetilde{\beta}_{-1}(\chi, \zeta) f(\widetilde{h})(1-\widetilde{h})\left(\frac{1}{\xi}\right)^{\frac{5}{8}-\widetilde{h}}=-\frac{3}{\widetilde{\beta}_{0}^{4}} \frac{\widetilde{\beta}_{-1}(\chi, \zeta)}{\xi^{\frac{5}{8}-\widetilde{h}}}
$$

Solving the obtained equation numerically, we obtain the following valued for parameter $\widetilde{h}: \widetilde{h}=0.0699928$. The coefficient $\widetilde{\beta}_{1}(\chi, \zeta)$, which depends on the both parameters $\chi$ and $\zeta$, could not be found without general numerical solution.

In order to find the second part of the $\widetilde{m}$-expansion it is necessary to consider the zero-toughness case, namely, the near-field $\left(x \ll \ell_{m \widetilde{m}}\right)$ of the $\widetilde{m} m$-edge solution. Here we neglect the pressure-dependent term in the right-hand side of the Eq. (C 1) because of the near-field of this edge solution is represented by $\widetilde{m}$-vertex solution that could potentially occur in the general solution when the leak-off process becomes pressureindependent (described by Carter's law). This asymptotic solution is derived by Garagash et al. (2011).

By using the aforesaid to limiting pats, we obtain the $\widetilde{m}$-expansion. 
Let us consider case of the large value of $\chi$ parameter and coordinate range $x \ll$ $\ell_{\widetilde{m} m}$. The $O(1)$ solution is located on the zero-storage face $(\widetilde{m} \widetilde{o} k)$. The next-order term corresponds to a small storage $\chi^{-1} \ll 1$ perturbation. This fact is the consequence of the lubrication equation written in the $\ell_{\widetilde{m} k}$ scaling $\left(\eta=\xi \chi^{2}\right)$ :

$$
\eta \frac{d \bar{\Pi}}{d \eta}-\frac{3 \bar{\Pi}}{8}=\left(\frac{\eta^{1 / 8}}{\chi}\right) \frac{1}{\bar{\Omega}^{2}}+\frac{1}{\bar{\Omega}^{3}}+\frac{\zeta^{3}}{\bar{\Omega}^{3} \eta^{1 / 2}} \int_{0}^{\eta} \frac{\bar{\Pi}\left(\eta^{\prime}\right) \eta^{\prime-3 / 8}}{2 \sqrt{\eta-\eta^{\prime}}} d \eta^{\prime}
$$

The first term in the right-hand side of the previous equation is a small storage correction at distances $\eta \ll \chi^{8}$ (or in the dimensional form $x \ll \ell_{\widetilde{m} m}$ ). As a result, the next-order term in the solution for the large $\chi$ parameter could be found in the form of Taylor expansion in the small storage parameter $\epsilon=\chi^{-1} \ll 1$ :

$$
\bar{\Omega}=\bar{\Omega}^{(0)}+\epsilon \bar{\Omega}^{(1)}, \bar{\Pi}=\bar{\Pi}^{(0)}+\epsilon \bar{\Pi}^{(1)}
$$

where $\bar{\Omega}^{(0)}, \bar{\Pi}^{(0)}$ is zero-storage $\widetilde{m} k$-edge solution.

Substituting the Taylor expansion into lubrication equation and keeping terms of order $O(\epsilon)$, we obtain the following equation:

$$
\begin{gathered}
\eta \frac{d \bar{\Pi}^{(1)}}{d \eta}-\frac{3 \bar{\Pi}^{(1)}}{8}=\frac{\eta^{1 / 8}}{\bar{\Omega}^{(0) 2}}-\frac{3 \bar{\Omega}^{(1)}}{\bar{\Omega}^{(0) 4}}- \\
-\frac{3 \bar{\Omega}^{(1)}}{\bar{\Omega}^{(0) 4}} \frac{\zeta^{3}}{\sqrt{\eta}} \int_{0}^{\eta} \frac{\bar{\Pi}^{(0)}\left(\eta^{\prime}\right) \eta^{\prime-3 / 8}}{2 \sqrt{\eta-\eta^{\prime}}} d \eta^{\prime}+\frac{1}{\bar{\Omega}^{(0) 3}} \frac{\zeta^{3}}{\sqrt{\eta}} \int_{0}^{\eta} \frac{\bar{\Pi}^{(1)}\left(\eta^{\prime}\right) \eta^{\prime-3 / 8}}{2 \sqrt{\eta-\eta^{\prime}}} d \eta^{\prime}
\end{gathered}
$$

In the far-field $\left(x \gg \max \left(\ell_{\widetilde{m} \widetilde{o}}, \ell_{\widetilde{m} k}\right)\right)$ the $O(1)$ term is given by the following equations:

$$
\bar{\Omega}(\xi)=\widetilde{\beta}_{0}+\frac{\check{\widetilde{\beta}}_{-3}}{\eta^{3 / 8}}+\frac{\widetilde{\beta}_{-1}(\chi, \zeta)}{\eta^{\frac{5}{8}-\widetilde{h}}}, \bar{\Pi}(\xi)=\widetilde{\beta}_{0} f(5 / 8)+\frac{\stackrel{\circ}{\tilde{\beta}}_{-3} f(1 / 4)}{\eta^{3 / 8}}+\frac{\widetilde{\beta}_{-1}(\chi, \zeta)}{\eta^{\frac{5}{8}-\widetilde{h}}} f(\widetilde{h})
$$

where the values of coefficients and $\widetilde{h}$ are derived earlier. The next-order term is found in the form of monomial solution. Using the condition for the considered coordinate range $(\eta \gg 1)$, we could neglect both terms relating to the pressure-dependent leak-off because of the following reason: substituting the expression of $\bar{\Pi}^{(0)}$ into the first integral $\left(\sim \frac{1}{\sqrt{\eta}} \int_{0}^{\eta} \frac{\bar{\Pi}^{(0)}\left(\eta^{\prime}\right) \eta^{\prime-3 / 8}}{\sqrt{\eta-\eta^{\prime}}} d \eta^{\prime}\right)$, it is possible to derive that it is proportional to the $\eta$ in the negative exponent; on the other hand, as shown earlier, that the second integral $\left(\sim \frac{1}{\sqrt{\eta}} \int_{0}^{\eta} \frac{\bar{\Pi}^{(1)}\left(\eta^{\prime}\right) \eta^{\prime-3 / 8}}{\sqrt{\eta-\eta^{\prime}}} d \eta^{\prime}\right)$ also provides with the term with the negative exponent of coordinate when the function $\bar{\Pi}^{(1)}$ has the form of monomial solution. As a result, both terms contain coordinate $\eta$ in the negative exponent, and the equation could be simplified to the following:

$$
\eta \frac{d \bar{\Pi}^{(1)}}{d \eta}-\frac{3 \bar{\Pi}^{(1)}}{8}=\frac{\eta^{1 / 8}}{\bar{\Omega}^{(0) 2}}-\frac{3 \bar{\Omega}^{(1)}}{\bar{\Omega}^{(0) 4}}
$$

Further, we balance this equation with the help of the next-order term in the form: $\bar{\Omega}^{(1)}=\widetilde{\beta}_{1} \eta^{1 / 8}, \bar{\Pi}^{(1)}=\widetilde{\beta}_{1} f(3 / 4) \eta^{1 / 8}$ where the numerical value of the coefficient $\widetilde{\beta}_{1}$ is derived earlier.

Using the obtained result, we could conclude that $\widetilde{m}$-expansion is the sum of the far-field of the $\widetilde{m} \widetilde{o} k$ face solution and the first term of the near-field of the $\widetilde{m} m$-edge expansion. 
Repeating this analysis for the higher order terms $O\left(\epsilon^{2}\right), O\left(\epsilon^{3}\right)$, we could derive other terms from near-field $\widetilde{m} m$-edge expansion.

As a result, the $\widetilde{m}$-expansion in the $m k$-scaling (returning from "bar" variable to the original variables in the $m k$-scaling) has the following form:

$$
\begin{gathered}
\Omega(\xi)=\chi^{1 / 4} \xi^{5 / 8}\left(\widetilde{\beta}_{0}+\sum_{j=1}^{3} \widetilde{\beta}_{j}\left(\frac{\xi}{\chi^{6}}\right)^{j / 8}+\left(\frac{\zeta^{8}}{\xi \chi^{2}}\right)^{3 / 8} \widetilde{\widetilde{\beta}}_{-3}+\frac{\widetilde{\beta}_{-1}(\chi, \zeta)}{\left(\chi^{2} \xi\right)^{\frac{5}{8}-\widetilde{h}}}\right) \\
\Pi(\xi)=\chi^{1 / 4} \xi^{-3 / 8}\left(\widetilde{\beta}_{0}+\sum_{j=1}^{2} \widetilde{\delta}_{j}\left(\frac{\xi}{\chi^{6}}\right)^{j / 8}+\frac{\widetilde{\beta}_{3}}{4 \pi}\left(\frac{\xi}{\chi^{6}}\right)^{3 / 8} \ln \left(\frac{\xi \chi^{6}}{\widetilde{\xi}_{0}}\right)\right. \\
\left.+\left(\frac{\zeta^{8}}{\xi \chi^{2}}\right)^{3 / 8} \stackrel{\check{\delta}}{-3}+\frac{\widetilde{\delta}_{-1}(\chi, \zeta)}{\left(\chi^{2} \xi\right)^{\frac{5}{8}-\widetilde{h}}}\right)
\end{gathered}
$$

where we use coefficients $\widetilde{\delta}_{j}=\widetilde{\beta}_{j} f\left(\frac{5}{8}+\frac{j}{8}\right)$ for $j=0,1,2, \stackrel{\widetilde{\delta}}{-3}=\stackrel{\widetilde{\beta}}{-3}_{-3} f(1 / 4)$ and $\widetilde{\delta}_{-1}=$ $\widetilde{\beta}_{-1} f(\widetilde{h})$ are utilised.

\section{Appendix D. Numerical scheme}

The numerical method is an extension of the approach of Garagash et al. (2011). In the this section, we recount the main parts of the numerical algorithms and also highlight differences borne by the more general problem formulation in this study as compared to Garagash et al. (2011).

The coordinate range $0<\xi<\infty$ is divided into three parts: $\left[0, \Xi_{0}\right],\left[\Xi_{0}, \Xi_{\infty}\right],\left[\Xi_{\infty},+\infty\right]$ (the normalized problem formulation in the $m k$-scaling is utilised). The first and the last segments are approximated by the analytical asymptotic expressions for the nearfield $\Pi_{0}^{*}(\xi)$ (subsection 3.3.1) and the far-field $\Pi_{\infty}^{*}(\xi)$ (subsection 3.3.2) correspondingly. For function $\Pi_{0}^{*}(\xi)$ (in the majority cases) we utilise only the leading term of the nearfield asymptotic expansion. However, when parameter $\zeta$ is small $(\zeta<1)$ we also use the next order power term that is derived in Appendix B. In turn, for function $\Pi_{\infty}^{*}(\xi)$, the $m$-vertex solution is utilised.

The intermediate segment, $\Xi_{0} \leqslant \xi \leqslant \Xi_{\infty}$, is the computational domain that is discretised by $n$ nodes into $n-1$ sub-intervals $\left(\xi_{i}, \xi_{i+1}\right), i=1, \ldots, n-1$ where $\xi_{1}=\Xi_{0}$ and $\xi_{n}=\Xi_{\infty}$. The value of pressure between nodes is approximated by linear combination of the constant function $\Pi_{0}(\xi)=-1$ and the far-field vertex solution $\Pi_{\infty}$. The whole representation of the pressure profile is the following:

$$
\Pi(\xi)= \begin{cases}\Pi_{0}^{*}(\xi), & \xi \in\left(0, \xi_{1}\right), \\ a_{i} \Pi_{0}(\xi)+b_{i} \Pi_{\infty}(\xi), & \xi \in\left(\xi_{i}, \xi_{i+1}\right), i=1, \ldots, n-1, \\ \Pi_{\infty}^{*}(\xi), & \xi \in\left(\xi_{n}, \infty\right)\end{cases}
$$

Coefficients $a_{i}$ and $b_{i}$ for $i=1, \ldots, n-1$ are found from values of pressure at nodes $\left(\Pi_{i}=\Pi\left(\xi_{i}\right)\right)$ by imposing continuity of pressure distribution:

$$
a_{i}=\frac{\Pi_{\infty}\left(\xi_{i}\right) \Pi_{i+1}-\Pi_{\infty}\left(\xi_{i+1}\right) \Pi_{i}}{\Pi_{\infty}\left(\xi_{i}\right) \Pi_{0}\left(\xi_{i+1}\right)-\Pi_{\infty}\left(\xi_{i+1}\right) \Pi_{0}\left(\xi_{i}\right)}, b_{i}=-\frac{\Pi_{0}\left(\xi_{i}\right) \Pi_{i+1}-\Pi_{0}\left(\xi_{i+1}\right) \Pi_{i}}{\Pi_{\infty}\left(\xi_{i}\right) \Pi_{0}\left(\xi_{i+1}\right)-\Pi_{\infty}\left(\xi_{i+1}\right) \Pi_{0}\left(\xi_{i}\right)}
$$

Moreover, in the end nodes we define value of pressure by using analytical asymptotic 
expansions $\Pi_{0}^{*}(\xi)$ and $\Pi_{\infty}^{*}(\xi)$ :

$$
\Pi_{1}=\Pi_{0}^{*}\left(\xi_{1}\right), \Pi_{n}=\Pi_{\infty}^{*}\left(\xi_{n}\right)
$$

Fracture opening is found by integrating the inverted elasticity integral, Eq. (2.3), using the above approximate representation for the net-pressure, in the following form

$$
\begin{aligned}
& \Omega(\xi)=\sqrt{\xi}+F\left[\Pi_{0}^{*}\right]\left(\xi, \xi_{1}\right)+\sum_{i=1}^{n-1}\left(a_{i} F\left[\Pi_{0}\right](\xi, \eta)+\right. \\
& \left.+b_{i} H\left[\Pi_{\infty}\right](\xi, \eta)\right)\left.\right|_{\eta=\xi_{i}} ^{\eta=\xi_{i+1}}+H\left[\Pi_{\infty}^{*}\right]\left(\xi, \xi_{n}\right)
\end{aligned}
$$

where $F[]$ and $H[]$ are integral operators defined by

$$
F[\Pi](\xi, \eta)=\frac{4}{\pi} \int_{0}^{\eta} K(\xi, \eta) \Pi(\eta) d \eta, H[\Pi](\xi, \eta)=\frac{4}{\pi} \int_{\eta}^{\infty} K(\xi, \eta) \Pi(\eta) d \eta
$$

The analytical expressions for the integrals in $F$ and $H$ for power and constant pressure functions that represent $\Pi_{0}^{*}(\xi), \Pi_{0}(\xi), \Pi_{\infty}^{*}(\xi)$ and $\Pi_{\infty}(\xi)$ are given in (Garagash and Detournay 2005; Garagash et al. 2011).

Further, in each node we write out the lubrication and elasticity equations, and together with boundary conditions for $\Pi_{1}$ and $\Pi_{n}$ they constitute the system of nonlinear algebraic equations. It is solved numerically by using Levenberg-Marquardt algorithm implemented in the SciPy library (Jones et al. 2001) of Python programming language.

\section{REFERENCES}

D.A. Spence and D.L. Turcotte. Magma-driven propagation of cracks. J. Geophys. Res., 90: $575-580,1985$.

J. R. Lister and R. C. Kerr. Fluid-mechanical models of crack propagation and their applications to magma transport in dykes. J. Geophys. Res., 96(B6):10,049-10,077, 1991.

A. M. Rubin. Tensile fracture of rock at high confining pressure: Implications for dike propagation. J. Geophys. Res., 98(B9):15,919-15,935, 1993.

D. T. Secor. Role of fluid pressure in jointing. American Journal of Science, 263:633-646, 1965. hydraulic cracks.

T. Engelder and A. Lacazette. Natural hydraulic fracturing. In C. Barton and O. Stephansson, editors, Rock Joints, pages 35-43, Rotterdam, 1990. Balkema.

E. Detournay. Mechanics of hydraulic fractures. Annu. Rev. Fluid Mech., 48(311-339), 2016. .

J. R. Lister. Buoyancy-driven fluid fracture: The effects of material toughness and of lowviscosity precursors. J. Fluid Mech., 210:263-280, 1990.

J. Desroches, E. Detournay, B. Lenoach, P. Papanastasiou, J. R. A. Pearson, M. Thiercelin, and A. H-D. Cheng. The crack tip region in hydraulic fracturing. Proc. Roy. Soc. London, Serie A(447):39-48, 1994.

D. I. Garagash and E. Detournay. The tip region of a fluid-driven fracture in an elastic medium. ASME J. Appl. Mech., 67(1):183-192, 2000.

D. I. Garagash, E. Detournay, and J. I. Adachi. Multiscale tip asymptotics in hydraulic fracture with leak-off. J. Fluid Mech., 669:260-297, 2011. .

E. Siebrits and A. P. Peirce. An efficient multi-layer planar 3D fracture growth algorithm using a fixed mesh approach. Int. J. Numer. Meth. Engng., 53:691-717, 2002.

A. Peirce and E. Detournay. An implicit level set method for modeling hydraulically driven fractures. Computer Meth. Appl. Mech. Eng., 197:2858-2885, 2008.

E. V. Dontsov and A. Peirce. A multiscale implicit level set algorithm (ILSA) to model hydraulic fracture propagation incorporating combined viscos, toughness, and leak-off asymptotics. Computer Meth. Appl. Mech. Eng., 313:53-84, 2017. 
H. Zia and B. Lecampion. Explicit versus implicit front advancing schemes for the simulation of hydraulic fracture growth. Int. J. Num. Anal. Meth. Geomech., 43:1300-1315, 2018.

A. A. Osiptsov. Fluid mechanics of hydraulic fracturing: a review. J. Petroleum Sci. Eng., 156: 513-535, 2017.

D. A. Spence and P. W. Sharp. Self-similar solution for elastohydrodynamic cavity flow. Proc. Roy. Soc. London, Ser. A(400):289-313, 1985.

B. Lenoach. The crack tip solution for hydraulic fracturing in a permeable solid. J. Mech. Phys. Solids, 43(7):1025-1043, 1995.

E. Detournay and D. Garagash. The tip region of a fluid-driven fracture in a permeable elastic solid. J. Fluid Mech., 494:1-32, 2003.

Y. Kovalyshen. Fluid-Driven Fracture in Poroelastic Medium. $\mathrm{PhD}$ thesis, University of Minnesota, 2010.

E. V. Dontsov. Tip region of a hydraulic fracture driven by a laminar-to-turbulent fluid flow. J. Fluid Mech., 797:R2, 2016.

B. Lecampion and H. Zia. Slickwater hydraulic fracture propagation: near-tip and radial geometry solutions. J. Fluid Mech., page in print, 2019.

M. Wrobel, G. Mishuris, and A. Piccolroaz. Energy release rate in hydraulic fracture: Can we neglect an impact of the hydraulically induced shear stress? Int. J. Eng. Sci., 111:28-51, 2017.

F.-E. Moukhtari and B. Lecampion. A semi-infinite hydraulic fracture driven by a shear-thinning fluid. J. Fluid Mech., 838:573-605, 2018.

E. V. Dontsov and O. Kresse. A semi-infinite hydraulic fracture with leak-off driven by a power-law fluid. J. Fluid Mech., 837:210-229, 2018.

E.D. Carter. Optimum fluid characteristics for fracture extension. In G.C. Howard and C.R. Fast, editors, Drilling and Production Practices, pages 261-270. American Petroleum Institute, Tulsa OK, 1957.

B. Carrier and S. Granet. Numerical modeling of hydraulic fracture problem in permeable medium using cohesive zone model. Engng. Fract. Mech., 79:312-328, 2012.

E. Sarris and P. Papanastasiou. The influence of the cohesive process zone in hydraulic fracturing modelling. Int. J. Fracture, 167:33-45, 2011. .

S. V. Golovin and A. N. Baykin. Influence of pore pressure on the development of a hydraulic fracture in poroelastic medium. Int. J. Rock Mech. Min. Sci., 108:198-208, 2018.

Y. Kovalyshen and E. Detournay. Propagation of a semi-infinite hydraulic fracture in a poroelastic medium. In Poromechanics V: Proceedings of the 5th Biot Conference on Poromechanics, pages 431-437, 2013.

J. R. Rice. Mathematical analysis in the mechanics of fracture. In H. Liebowitz, editor, Fracture, an Advanced Treatise, volume II, chapter 3, pages 191-311. Academic Press, New York NY, 1968.

G. K. Batchelor. An Introduction to Fluid Dynamics. Cambridge University Press, Cambridge UK, 1967.

G. R. Irvin. Analysis of stresses and strains near the end of a crack traversing a plate. ASME J. Appl. Mech., 24:361-364, 1957.

B.A. Bilby and J.D. Eshelby. Dislocations and the theory of fracture. In H. Liebowitz, editor, Fracture, an Advanced Treatise, volume I, chapter 2, pages 99-182. Academic Press, New York NY, 1968.

M. R. Chandler, P. G.Meredith, N. Brantut, and B. R. Crawford. Fracture toughness anisotropy in shale. J. Geophys. Res., 121(3):1706-1729, 2016.

A. Li, W. Ding, J. He, P. Dai, S. Yin, and F. Xie. Investigation of pore structure and fractal characteristics of organic-rich shale reservoirs: A case study of lower cambrian qiongzhusi formation in malong block of eastern yunnan province, south china. Marine and Petroleum Geology, 70:46-57, 2016.

K. Magara. Comparison of porosity-depth relationships of shale and sandstone. J. Petroleum Geol., 3(2):175-185, 1980.

G. E. Manger. Porosity and bulk density of sedimentary rocks. Technical Report 1144, USGS, 1963.

J. B. Walsh. Effect of pore pressure and confining pressure on fracture permeability. Int. J. Rock Mech. Min. Sci. \&6 Geomech. Abstr., 18(5):429-435, 1981. 
A. Peirce. Modeling multi-scale processes in hydraulic fracture propagation using the implicit level set algorithm. Comput. Methods Appl. Mech. Engrg., 283:881-908, 2015.

H. Carslaw and J. C. Jaeger. Conduction of Heat in Solids. Oxford University Press, 2nd edition, 1959.

D. I. Garagash and E. Detournay. Plane-strain propagation of a fluid-driven fracture: Small toughness solution. ASME J. Appl. Mech., 72(6):916-928, 2005.

E. Jones, T. Oliphant, and P. Peterson. SciPy: Open source scientific tools for Python. 2001. URL http://www.scipy.org/. 